\title{
A Mechanism Design Based Approach to Solving Parking Slot Assignment in the Information Era
}

\author{
Bo Zou ${ }^{1 *}$, Nabin Kafle ${ }^{1}$, Ouri Wolfson ${ }^{2}$, Jie (Jane) Lin $^{1}$ \\ ${ }^{1}$ Department of Civil and Materials Engineering, University of Illinois at Chicago, IL, USA \\ ${ }^{2}$ Department of Computer Science, University of Illinois at Chicago, IL, USA
}

\begin{abstract}
This paper proposes a mechanism design based approach for public parking slot assignment in an environment empowered by recent advances in parking sensing, infrastructure-to-vehicle, and vehicleto-infrastructure communications. An important part of the parking slot assignment deals with eliciting truthful private information from drivers while maximizing social welfare. We consider both static and dynamic mechanisms and provide theoretic proofs that, by using coupled slot allocation and payment rules, drivers will be incentivized to participate in the assignment process and truthfully report their private information. The parking manager will benefit by generating non-negative revenue from each assigned driver. Our numerical analysis provides further insights into the implementation of the dynamic mechanisms.
\end{abstract}

Keywords: parking slot assignment, mechanism design, truth telling, allocation rule, payment

* Corresponding author.

Email addresses: bzou@uic.edu (B. Zou), nkafle3@uic.edu (N. Kafle), wolfson@cs.uic.edu (O. Wolfson), janelin@uic.edu (J. Lin). 


\section{Introduction}

Parking presents a major challenge in metropolitan areas, where the supply of physical parking infrastructure is often constrained by available land vis-à-vis the extent of business, commercial, and other activities. As a result, arriving drivers spend many minutes searching for available parking space, which constitutes an important yet often ignored source of congestion. Previous research concludes that the average search time for parking in large cities is $8.1 \mathrm{~min}$ per vehicle; in addition, $30 \%$ of city road traffic is cruising for parking (Shoup, 2006). Putting this in aggregate, parking space search in the city of Chicago resulted in 63 million vehicle-miles-traveled, 3.1 million gallons of gasoline consumption, and 48,000 tons of $\mathrm{CO}_{2}$ emissions per year (Ayala et al., 2011). Given that the possibility of increasing parking supply is limited, solving the urban parking problem often requires a demand management perspective.

Allocation of parking resources using existing infrastructure is being made increasingly efficient thanks to recent technological advances in sensing to collect real-time information on parking availability (Park et al., 2008, Panja et al., 2011) and infrastructure-to-vehicle communications to disseminate parking availability information to drivers. Examples of the latter are: data access via a peer-to-peer (P2P) environment (Wolfson et al., 2004; Kokolaki et al., 2011); coupled open space detection with information sharing (Mathur et al., 2010); and wireless ad-hoc networking for data sharing (Verroios et al., 2011). These new technologies enable real-time allocation of parking slots to demanding drivers.

New vehicle-to-infrastructure communication technologies further allow private information from drivers to be sent to the parking manager. For a driver, her parking-related information can include how much she values parking, when she arrives, how late she could stay and wait to be assigned, and the time of departure from the parking. Smartphone applications today have made information submission from drivers to the parking manager in real time and at almost no cost. Exchange of information on parking availability and demanding drivers between the parking manager and drivers promotes the development of disaggregate, agent-based parking slot assignment. A public parking manager, for example, can assign parking slots based on the information he has to achieve desired system-wide outcomes, such as maximizing social welfare.

A number of recent studies have looked into parking management in the context of a centralized authority and with information technologies. The relationship between parking price and real-time parking occupancy is investigated in Qian and Rajagopal (2014) under user equilibrium. Qian and Rajagopal (2013) consider information provision and pricing jointly as an efficient way of managing parking traffic. Caicedo (2010) develops a demand assignment model to evaluate the benefits of manipulating information to reduce time and distance involved in search of parking space. Ayala et al. (2011) present a slot assignment model that maximizes social welfare and investigate the price of anarchy. In Ayala et al. (2012), a parking pricing authority uses information about parking availability and drivers' natural cost to set charges in order to entice drivers and minimize total vehicle driving distance. An auction-based and a vehicle-specific pricing algorithms are further proposed to move Nash equilibrium to system optimum.

In the above studies, the objective of a parking manager/pricing authority, if there exists, is to minimize system-wide parking-related costs. The costs are calculated based on drivers' distance to the parking garage, parking price, and in some cases cruising time for parking. However, the heterogeneity of parking benefits across drivers has rarely been considered. Different drivers also have non-identical value-of-travel-time and thus the cost of driving/cruising for the same amount of time will be different. From the economic efficiency perspective, the best assignment should give available parking slots to drivers who value the slots most.

One fundamental assumption underlying centralized parking slot assignment problems is that truthful information of drivers is readily available to the parking manager. However, having the truthful private information relies on the goodwill of drivers who fully cooperate with the parking manager. The reality is 
often different, since drivers are inherently selfish. With knowledge about how slots are assigned and information on parking slot availability, a driver can misreport private information in order to maximize her own utility. Given untruthful driver private information, suboptimal system-wide allocation will result. To prevent this, appropriate mechanisms must be in place that incentivize drivers to report truthful private information. Such mechanisms need to align the selfish behavior of drivers with the system objective, so that optimal allocation of parking resources can be achieved under an intervened driver equilibrium.

This paper contributes to the parking literature by introducing mechanism design principles to parking management in an environment empowered by parking sensing, infrastructure-to-vehicle, and vehicle-toinfrastructure communications. Both drivers and the parking manager will be informed of parking slot availability in real time. The parking facility in our study is publicly owned, with the objective of the parking manager being maximizing social welfare, which is characterized by the sum of drivers' net utilities gained from using the parking facility. We consider both static and dynamic parking slot assignments, and show that truth telling is unlikely to hold absent intervention due to the intrinsic selfishness of drivers. We develop mechanisms that blend slot allocation with pricing rules such that drivers' utility-maximizing decisions are incentive compatible, i.e., revealing their true information is the best action to take for each driver. ${ }^{1}$ The mechanisms developed in this study fall into the category of "direct mechanisms", in which drivers are asked to reveal their preference parameters (i.e., valuation). In contrast to this are indirect mechanisms (Nisan et al., 2007), which this paper does not deal with. The pricing scheme in our mechanisms aligns drivers' selfish behavior with the objective of the public parking manager, so that optimal allocation of parking resources is achieved. We show that implementing the proposed mechanisms can increase parking efficiency in the real world.

We begin with an overview of mechanism design in Section 2, followed by discussion on a static parking slot assignment mechanism in Section 3. The case of dynamic parking is presented in Section 4, where we describe the base mechanism, provide the formal proof that the mechanism is incentive compatible, and explore other properties of the mechanism and its variant. To further examine the dynamic parking slot assignment, numerical experiments are performed in Section 5. Section 6 offers conclusions and directions for future research.

\section{An overview of mechanism design with a simple example}

According to Hartline (2013), mechanism design gives a theory for the design of protocols, services, laws, or other "rules of interaction" in which selfish behavior leads to good outcomes. "Selfish behavior" means that each agent in the mechanism individually tries to maximize her own utility. Such behavior is defined as rational. "Leads" means in equilibrium. A set of agent strategies is in equilibrium if no agent wants to change her strategy unilaterally. "Good"-ness of an outcome is assessed with respect to the criteria or goals of the designer. A natural economic criterion is social welfare, the sum of utilities of all agents. An alternative criterion can be profit, which is the total payment made by agents to the mechanism less any cost of providing the mechanism. Therefore, mechanism design intends to align agents' best strategies under the rational behavior assumption with the achievement of good outcomes, as desired by the mechanism designer. Mechanism design is also viewed as reverse game theory, in which the outcome of the game is predefined and the agents are expected to take their strategies to attain the pre-defined outcome.

Research on mechanism design originates from the seminal work of William Vickery and James Mirrlees, on seemingly disparate issues where information asymmetries are a key component. Vickery's research concerns properties of different types of auctions, and how they can be best designed to generate economic efficiency (Vickery, 1961, 1962). Mirrlees (1971) extends Vickery's early formulation on income taxation design and finds the optimal income taxes. Because of their contribution to the theories of auctions, incentives, and income taxation, William Vickrey and James Mirrless were awarded the

\footnotetext{
${ }^{1}$ In this paper we interchangeably use the terms "truth telling" and "incentive compatible".
} 
Nobel Prize on Economics in 1996. Later in 2007, Leonid Hurwicz, Eric Maskin, and Roger Myerson jointly received the same Prize for their contribution to the theory of mechanism design. First applications of mechanism design appeared around 1993-1994, when the design and operation of the radio spectrum auctions was implemented in the US and then spread to the rest of the world (Milgrom, 2004; McMillan, 1994). Since then, mechanism design has been put into practice for auctions of electric power, $\mathrm{CO}_{2}$ abatement, timber, freight logistics, and various assets (Milgrom, 2004; Caplice and Sheffi, 2006).

In our context of parking slot assignment, the mechanism designer and agents correspond to the public parking manager and drivers respectively. In order to maximize social welfare from parking slot assignment, the parking manager needs to find out drivers' valuation (i.e., utility) for parking, which is $a$ priori only known to each driver him/herself. As the mechanism designer, the parking manager attempts to elicit such private, hidden information from drivers such that drivers, under an appropriate mechanism setting, find it their best interest to truthfully report their private information.

Before delving into detailed parking slot assignment mechanisms, we first demonstrate a simple, second price auction example to give the reader a conceptual understanding about mechanism design. The major purpose is to show that the equilibrium strategy of an agent is to express her true private value.

Consider an auction that two agents expressing their values (bidding) for a single item. The true values of the two agents are $b_{1}$ and $b_{2}$. The mechanism designer wants the agent with the higher valuation to get the item, which essentially maximizes social welfare in the system. To this end, the rule of the auction is that the agents with higher bid wins and pays the other agent's bid. Consequently the net utility (i.e., payoff) received by the winning agent is the difference between her true valuation and the price she pays. For illustration we focus on the strategy of agent 1 , and assume that agent 2 truthfully reports her value $b_{2}$. Agent 1 can express her bid $\widehat{b_{1}}$, which can be greater than, equal to or less than $b_{1}$. Without knowing the $b_{2}$, agent 1 will ponder over all possible cases:

1) $b_{1}>b_{2}$.

Table 1: Possible strategies of agent 1 and associated payoff when $b_{1}>b_{2}$

\begin{tabular}{|l|l|l|}
\hline Strategy for agent 1 & $\begin{array}{l}\text { Agent } 1 \text { 's payoff if she wins } \\
\left.\text { (if } \widehat{b_{1}} \geq b_{2}\right)^{2}\end{array}$ & $\begin{array}{l}\text { Agent 1's payoff if she loses } \\
\left.\text { (if } \widehat{b_{1}}<b_{2}\right)\end{array}$ \\
\hline $\operatorname{Bid} \widehat{b_{1}}=b_{1}$ & $b_{1}-b_{2}>0$ & Not possible \\
\hline $\operatorname{Bid} \widehat{b_{1}}>b_{1}$ & $b_{1}-b_{2}>0$ & Not possible \\
\hline $\operatorname{Bid} \widehat{b_{1}}<b_{1}$ & $b_{1}-b_{2}>0$ & 0 \\
\hline
\end{tabular}

In this case, the strategies $\widehat{b_{1}} \geq b_{1}$ result in equal outcome for agent 1 , and are superior to the strategy $\widehat{b_{1}}<b_{1}$, because the latter may result in zero net utility.

2) $b_{1}<b_{2}$.

Table 2: Possible strategies of agent 1 and associated payoff when $b_{1}<b_{2}$

\begin{tabular}{|l|l|l|}
\hline Strategy for agent 1 & $\begin{array}{l}\text { Agent } 1 \text { 's payoff if she wins } \\
\left.\text { (if } \widehat{b_{1}} \geq b_{2}\right)\end{array}$ & $\begin{array}{l}\text { Agent 1's payoff if she loses } \\
\left.\text { (if } \widehat{b_{1}}<b_{2}\right)\end{array}$ \\
\hline $\operatorname{Bid} \widehat{b_{1}}=b_{1}$ & Not possible & 0 \\
\hline $\operatorname{Bid} \widehat{b_{1}}>b_{1}$ & $b_{1}-b_{2}<0$ & 0 \\
\hline $\operatorname{Bid} \widehat{b_{1}}<b_{1}$ & Not possible & 0 \\
\hline
\end{tabular}

In this case, the strategies $\widehat{b_{1}} \leq b_{1}$ result in equal outcome for agent 1 , and are superior to the strategy $\widehat{b_{1}}>b_{1}$, because the latter may result in negative net utility.

\footnotetext{
${ }^{2}$ For simplicity, we assume that when $\widehat{b_{1}}=b_{2}$, the item is allocated to agent 1 .
} 
3) $b_{1}=b_{2}$. No matter what agent 1 bids and whether she gets the item, her utility is always zero. Therefore, agent 1 is indifferent to the three bidding strategies.

The above simple example makes it clear that the overall best strategy for agent 1 is to bid truthfully (i.e., $\widehat{b_{1}}=b_{1}$ ). Therefore, the mechanism of second price auction elicits truthful value reporting from agent 1 .

\section{Static parking case}

We start with discussion of static parking slot assignment in order to gain basic insights about how truth telling can be elicited through appropriate allocation and pricing schemes. The objective of the public parking manager is to maximize social welfare by allocating slots to drivers. We assume fixed cost associated with administration and wear-and-tear expenses of the parking infrastructure. Therefore, social welfare maximization is equivalent to matching drivers with available slots such that the sum of their received values from using the parking slots is maximized. Note that the parking assignment problem we consider here is general: it does not have to be all from the same parking facility (e.g., a parking garage). The model framework also applies to assigning parking slots from multiple facilities to drivers - as long as all slots are under the control of a single public authority.

To set up, let us consider a public parking system which has $J$ available slots to accommodate demand from a maximum of $I$ drivers. Each driver has the following utility form:

$$
u_{i}(\boldsymbol{a})=\sum_{j=1}^{J} v_{i j}(\boldsymbol{a}) a_{i j}-p_{i}
$$

where $v_{i j}$ is the positive valuation of driver $i$ from using parking slot $j ; p_{i}$ is the payment made by driver $i$; $a_{i j}$ is a $0-1$ indicator of whether slot $j$ is assigned to driver $i$. $\boldsymbol{a}$ is the vector of resource allocation results: $\boldsymbol{a}=\left(a_{11} \ldots, a_{i j}, \ldots, a_{I J}\right)$.

Because the parking manager attempts to maximize total welfare of all drivers by assigning slots, the allocation outcome $\boldsymbol{a}$ depends upon the valuation information submitted by drivers $v_{i j}$ 's. Each $v_{i j}$ could be considered as the net of multiple pieces of information, including for example the benefits received by driver $i$ from using parking slot $j$ and the associated cost (e.g., "ordinary" parking ticket price, as is charged in real world systems without imposing mechanism design), but excluding mechanism-specific payment $p_{i}$. Given the drivers' submitted valuation information, the optimal allocation $\boldsymbol{a}$ is obtained by solving the following integer program:

$$
\begin{aligned}
\boldsymbol{a}= & \operatorname{argmax}_{\boldsymbol{y} \in \boldsymbol{Y}} \sum_{i=1}^{I} \sum_{j=1}^{J} v_{i j}(\boldsymbol{y}) y_{i j} \\
\text { s.t. } & y_{i j}= \begin{cases}1 \text { if driver } i \text { is assigned to resource } j \\
0 \quad \text { otherwise }\end{cases} \\
& \sum_{j=1}^{J} y_{i j} \leq 1 \quad \forall i \in\{1, \ldots, I\} \\
& \sum_{i=1}^{I} y_{i j} \leq 1 \quad \forall j \in\{1, \ldots, J\}
\end{aligned}
$$

The objective function (2.1) solves for allocation outcome $\boldsymbol{y}=\left(y_{11}, \ldots y_{1 J}, \ldots, y_{I 1}, \ldots, y_{I J}\right)$ which comes from maximizing total social welfare. Each element $y_{i j}(i=1, \ldots, I ; j=1, \ldots, J)$ takes a $0-1$ value, indicating whether slot $j$ is allocated to driver $i$, as specified in (2.2). $\boldsymbol{Y}$ is the set of all possible assignments. In this paper, we consider the possibility of drivers deliberately misreporting their valuation $v_{i j}$, because doing so can give her higher net utility than telling the truth, assuming that they know the allocation and payment rules. Therefore, each driver's reported value $\boldsymbol{v}_{i}$ is determined based on the allocation and payment rules, which are implicitly reflected by the allocation outcome, i.e., $v_{i j}=v_{i j}(\boldsymbol{y})$. Constraint (2.3) assumes that each driver will be assigned at most one parking slot, and it is possible that one is not allocated a slot. Constraint (2.4) stipulates that a parking slot can be used by at most one driver. 
It is evident that program (2.1)-(2.4) alone, which intends to achieve system optimum allocation outcome, is different from each individual driver's selfish objective, which is to maximize her own net utility. In effect, the latter leads to gaming among the drivers. In our context, each driver understands the rule but does not know exactly the valuation of other drivers. Without other intervention, each driver may report her $\boldsymbol{v}_{i}$ to be an as large as possible value in order to increase her chance of getting the most desired slot, as doing so increases her importance in the allocation process. An appropriate payment scheme needs to be imposed together with the above allocation rule to ensure that the parking manager can induce truthful information from drivers while performing slot allocation and determining payment. In other words, individuals' selfish behavior, under the allocation scheme and with the payment, will be aligned with the parking manager's intent to maximize social welfare. It is worth mentioning that payment from drivers to the parking manager is only internal money transfer, and does not affect the calculation of overall social welfare. We consider the following payment scheme:

$$
p_{i}=\sum_{k \neq i} \sum_{j=1}^{J} v_{k j}(\boldsymbol{b}) b_{k j}-\sum_{k \neq i} \sum_{j=1}^{J} v_{k j}(\boldsymbol{a}) a_{k j}
$$

where $\boldsymbol{b}$ is obtained by solving a similar problem to (2) but driver $i$ is absent:

$$
\begin{aligned}
\boldsymbol{b}= & \operatorname{argmax}_{\boldsymbol{y} \in \boldsymbol{Y}} \sum_{k \neq i} \sum_{j=1}^{J} v_{k j}(\boldsymbol{y}) y_{k j} \\
\text { s.t. } & y_{k j}=\left\{\begin{array}{rr}
1 \text { if driver } k \text { is assigned to resource } j \\
0
\end{array}\right. \\
& \sum_{j=1}^{J} y_{k j} \leq 1 \quad \forall k \in\{1, \ldots, i-1, i+1, \ldots, I\} \\
& \sum_{k \neq i} y_{k j} \leq 1 \quad \forall j \in\{1, \ldots, J\}
\end{aligned}
$$

Clearly, $\boldsymbol{b}$ does not depend on driver $i$ 's reporting $\boldsymbol{v}_{i}$. The first term of the right hand side of (3), $\sum_{k \neq i} \sum_{j=1}^{J} v_{k j}(\boldsymbol{b}) b_{k j}$, can be viewed by driver $i$ as a constant, no matter what she reports to the parking manager about her own valuation $\boldsymbol{v}_{i}$. As shown in (4.1)-(4.4), this term gives the maximum social welfare from parking slot allocation when $i$ does not participate. Note that the allocation outcome $\boldsymbol{b}$ still involves driver $i$ (because $\boldsymbol{b} \in \boldsymbol{Y}$ ), but the allocation of driver $i$ is arbitrary after total welfare of the other drivers is maximized. When no slot is left after maximizing total welfare of the other drivers, $b_{i j}=0 \forall j$. The second summation term on the right hand side of (3), $\sum_{k \neq i} \sum_{j=1}^{J} v_{k j}(\boldsymbol{a}) a_{k j}$, represents the maximum social welfare of all drivers other than $i$, when $i$ partakes in the parking slot assignment. As a consequence, $p_{i}$ can be interpreted as the externality caused by driver $i$ to all other drivers. In the special case that driver $i$ is not assigned a slot in $\boldsymbol{a}$, the allocation outcome for all other drivers will be the same in $\boldsymbol{a}$ and $\boldsymbol{b}: \sum_{k \neq i} \sum_{j=1}^{J} v_{k j}(\boldsymbol{b}) b_{k j}=\sum_{k \neq i} \sum_{j=1}^{J} v_{k j}(\boldsymbol{a}) a_{k j}$. Consequently no payment will incur to the driver. Substituting (3) for (1), the utility received by driver $i$ is equal to:

$$
u_{i}(\boldsymbol{a})=\sum_{i=1}^{I} \sum_{j=1}^{J} v_{i j}(\boldsymbol{a}) a_{i j}-\sum_{k \neq i} \sum_{j=1}^{J} v_{k j}(\boldsymbol{b}) b_{k j}
$$

where the first term is the maximum social welfare which is achieved under allocation $\boldsymbol{a}$. Note that $\sum_{k \neq i} \sum_{j=1}^{J} v_{k j}(\boldsymbol{b}) b_{k j}$ is independent of $\boldsymbol{v}_{i}$. Therefore, the payment scheme aligns each driver's utility maximization with the system welfare maximization objective. The combination of allocation rule (2) and payment scheme (3) comprises the parking slot assignment mechanism $\boldsymbol{M}(\boldsymbol{a}, \boldsymbol{p})$, where $\boldsymbol{p}=\left(p_{1}, \ldots, p_{I}\right)$. In Proposition 1 below, we show that mechanism $\boldsymbol{M}(\boldsymbol{a}, \boldsymbol{p})$ induces truth telling of drivers.

Proposition 1. Mechanism $\boldsymbol{M}(\boldsymbol{a}, \boldsymbol{p})$ induces truth telling of drivers. In other words, the drivers cannot be better off by not declaring their true valuation $\boldsymbol{v}$.

Proof. For a given driver $i$, we fix $\boldsymbol{v}_{-i}=\left(\boldsymbol{v}_{1}, \ldots, \boldsymbol{v}_{i-1}, \boldsymbol{v}_{i+1}, \ldots, \boldsymbol{v}_{I}\right)$. Consider two scenarios: in the first one driver $i$ submits her true valuation $\boldsymbol{v}_{i}$; in the second one a different valuation $\boldsymbol{v}_{i}^{\prime}$ is declared by 
driver $i$. The utility received by driver $i$ when reporting the truth is expressed in Equation (5). When $\boldsymbol{v}_{i}{ }_{i}$ is declared, the utility received by driver $i$ would become

$$
u_{i}\left(\boldsymbol{a}^{\prime}\right)=\sum_{i=1}^{I} \sum_{j=1}^{J} v_{i j}\left(\boldsymbol{a}^{\prime}\right) a_{i j}^{\prime}-\sum_{k \neq i} \sum_{j=1}^{J} v_{k j}(\boldsymbol{b}) b_{k j}
$$

where allocation $\boldsymbol{a}^{\prime}$ comes from solving the following problem:

$$
\begin{aligned}
& \boldsymbol{a}^{\prime}=f\left(\boldsymbol{v}_{i}^{\prime}, \boldsymbol{v}_{-i}\right)=\operatorname{argmax}_{\boldsymbol{y} \in \boldsymbol{Y}}\left(\sum_{j=1}^{J} \boldsymbol{v}_{i j}^{\prime}(\boldsymbol{y}) y_{i j}+\sum_{k \neq i} \sum_{j=1}^{J} v_{k j}(\boldsymbol{y}) y_{k j}\right) \\
& \text { s.t. } y_{i j}=\left\{\begin{array}{lr}
1 \text { if driver } i \text { is assigned to resource } j \\
0 & \text { otherwise }
\end{array} \quad \forall i \in\{1, \ldots, I\} ; j \in\{1, \ldots, J\}\right. \\
& \sum_{j=1}^{J} y_{i j} \leq 1 \quad \forall i \in\{1, \ldots, I\} \\
& \sum_{i=1}^{I} y_{i j} \leq 1 \quad \forall j \in\{1, \ldots, J\}
\end{aligned}
$$

Recall that $\boldsymbol{a}$ represents the best parking allocation outcome with true valuation: $\sum_{i=1}^{I} \sum_{j=1}^{J} v_{i, j}(\boldsymbol{a}) a_{i j} \geq \sum_{i=1}^{I} \sum_{j=1}^{J} v_{i, j}\left(\boldsymbol{a}^{\prime}\right) a_{i j}^{\prime}$. Because of this and the fact that $\sum_{k \neq i} \sum_{j=1}^{J} v_{k, j}(\boldsymbol{b}) b_{k j}$ is a common term in (5) and (6) and only a constant for driver $i, u_{i}(\boldsymbol{a}) \geq u_{i}\left(\boldsymbol{a}^{\prime}\right)$, i.e., reporting the true valuation is the best thing to do for driver $i$. The same is for all other drivers. Therefore, mechanism $\boldsymbol{M}(\boldsymbol{a}, \boldsymbol{p})$ induces truth-telling.

We have hence shown that the net utility of drivers when expressing their true valuation is no less than the net utility while expressing any other valuation. Indeed, this holds true even when other drivers report untrue valuations. The induced game then has a (weakly) dominant strategy equilibrium. Mechanism $\boldsymbol{M}(\boldsymbol{a}, \boldsymbol{p})$ is dominant strategy incentive compatible (DSIC): irrespective of what other drivers report, it is always best for a driver to express her true value.

The objective of maximizing individual utility implicitly assumes that each driver behaves rationally. Another aspect of being rational is that drivers can opt out if one has to pay a higher price than the valuation gained from using the slot. A nice property of mechanism $\boldsymbol{M}(\boldsymbol{a}, \boldsymbol{p})$ is that each driver always receives non-negative utility with the payment. In addition, under $\boldsymbol{M}(\boldsymbol{a}, \boldsymbol{p})$ drivers never receive money from the parking manager, i.e., the parking authority is guaranteed to collect non-negative revenue from the users. Below we summarize the properties and provide brief proofs.

Proposition 2. All drivers are willing to participate in the parking slot assignment under $\boldsymbol{M}(\boldsymbol{a}, \boldsymbol{p})$, as $\boldsymbol{M}(\boldsymbol{a}, \boldsymbol{p})$ gives drivers non-negative utility.

Proof. Recall from (5) that for driver $i=1, \ldots, I$ :

$$
\begin{aligned}
u_{i}(\boldsymbol{a}) & =\sum_{i=1}^{I} \sum_{j=1}^{J} v_{i j}(\boldsymbol{a}) a_{i j}-\sum_{k \neq i} \sum_{j=1}^{J} v_{k j}(\boldsymbol{b}) b_{k j} \\
& \geq \sum_{i=1}^{I} \sum_{j=1}^{J} v_{i j}(\boldsymbol{a}) a_{i j}-\sum_{k \neq i} \sum_{j=1}^{J} v_{k j}(\boldsymbol{b}) b_{k j}-\sum_{j=1}^{J} v_{i j}(\boldsymbol{b}) b_{i j} \\
& =\sum_{i=1}^{I} \sum_{j=1}^{J} v_{i j}(\boldsymbol{a}) a_{i j}-\sum_{i=1}^{I} \sum_{j=1}^{J} v_{i j}(\boldsymbol{b}) b_{i j} \geq 0
\end{aligned}
$$

In the second line above, driver $i$ will be assigned to any remaining slot available as mentioned before (if no slot is left, then $b_{i j}=0 \forall j$ ). Given $v_{i j}$, allocation outcome $\boldsymbol{a}$ maximizes the overall welfare. Therefore, $\sum_{i=1}^{I} \sum_{j=1}^{J} v_{i j}(\boldsymbol{a}) a_{i j} \geq \sum_{i=1}^{I} \sum_{j=1}^{J} v_{i j}(\boldsymbol{b}) b_{i j}$. In other words, each driver always receives nonnegative utility with $\boldsymbol{M}(\boldsymbol{a}, \boldsymbol{p})$.

The non-negative utility resulting from implementing $\boldsymbol{M}(\boldsymbol{a}, \boldsymbol{p})$ implies that drivers will have the incentive to partake in the parking slot assignment process. On the other hand, the parking manager should also be willing to implement $\boldsymbol{M}(\boldsymbol{a}, \boldsymbol{p})$, if non-negative revenue can be generated. This is indeed the case, as shown in Proposition 3.

Proposition 3. The parking manager always collects non-negative revenue from each of the participating drivers under $\boldsymbol{M}(\boldsymbol{a}, \boldsymbol{p})$. 
Proof. Recall that the amount paid by driver $i$ under $\boldsymbol{M}(\boldsymbol{a}, \boldsymbol{p})$ is: $p_{i}=\sum_{k \neq i} \sum_{j=1}^{J} v_{k j}(\boldsymbol{b}) b_{k j}-$ $\sum_{k \neq i} \sum_{j=1}^{J} v_{k j}(\boldsymbol{a}) a_{k j}$, where $\boldsymbol{b}=\operatorname{argmax}_{\boldsymbol{y} \in \boldsymbol{Y}} \sum_{k \neq i} \sum_{j=1}^{J} v_{k j}(\boldsymbol{y}) y_{k j}$. Therefore $\sum_{k \neq i} \sum_{j=1}^{J} v_{k j}(\boldsymbol{b}) b_{k j} \geq$ $\sum_{k \neq i} \sum_{j=1}^{J} v_{k j}(\boldsymbol{a}) a_{k j}$, i.e., $p_{i} \geq 0$.

The proposed static parking slot allocation mechanism is, in effect, a generalization of the VickeryClarke-Grove (VCG) mechanism with Clarke pivotal rule (Nisan, 2007) in that our setting allows the most general valuation reporting: each driver can value each parking slot differently and consequently report $J$ distinct values. Like the VCG mechanism, the price-to-pay for each driver in order to induce truth telling is equal to the externality the driver imposes on her peers, when the allocation objective is to maximize social welfare. This very general heterogeneous valuation is plausible when the parking slot assignment is performed in a reserved or other similar setting, where drivers have sufficient time to figure out their true valuation for different parking places in a parking facility. On the other hand, when the decision time is limited, as is often the case in real-time parking, it is more likely that each driver comes up with a single value for being able to park. In this case, the static parking slot allocation mechanism will collapse to the classic VCG mechanism. The remaining part of this study focuses on the real-time parking case. However, the fact that drivers come and go dynamically makes direct application of the static mechanism to the dynamic case not incentive compatible. New mechanisms need to be devised to elicit truthful information from drivers.

\section{Dynamic parking case}

\subsection{The mechanism}

In the dynamic setting, we consider that the parking manager would ask drivers to report not only their valuation but also time related information, such as when to arrive and depart, and how long one can, after arrival, wait to be assigned a parking slot. We term the collective information the type of driver $i, \boldsymbol{\theta}_{i}=\left(e_{i}, w_{i}, d_{i}, v_{i}\right)$, where $e_{i}, w_{i}, d_{i}$ and $v_{i}$ denote respectively the reported arrival time, latest waiting, departure time, and slot valuation by driver $i$. Note that, as is argued at the end of Section 2, the driver's valuation will be better represented by a single scalar $v_{i}$ in the real-time dynamic setting. Similar to the static case, each $v_{i}$ should be interpreted as the net of benefits received by driver $i$ from parking and the associated cost (e.g., parking duration related cost) excluding mechanism-specific payment. We consider a time horizon, e.g., a day, for which the dynamic parking slot allocation is performed. Because of the discrete nature of the assignment problem, the time horizon is decomposed into a series of equal-length time intervals. At any given time, the parking manager will assign available slots to waiting drivers who submitted their type information and are waiting to be assigned a parking slot. Each driver only submits her type information once. We assume that the parking manager does not have information about future arrivals of drivers and their type information - the parking manager performs "myopic" assignment in each period based on the information he has received so far. ${ }^{3}$

It may be tempting to consider direct implementation of the static parking slot assignment mechanism $\boldsymbol{M}(\boldsymbol{a}, \boldsymbol{p})$, which collapses into VCG mechanism with Clark pivotal rule when $v_{i}$ is a scalar for each driver $i$, in each time period in the dynamic environment. Specifically, if $n$ vacant slots are to be assigned to more than $n$ waiting drivers at a period, then those $n$ drivers with $n$ highest valuation will each be given a slot; the corresponding payment would become identical for all assigned drivers and equal the $(n+1)^{\text {th }}$ highest valuation - the highest valuation among the unassigned drivers, which is the externality caused by an assigned driver (if an assigned driver were absent, then the driver with the $(n+1)^{\text {th }}$ highest valuation will be assigned to the vacated slot). However, truth telling would no longer hold. A driver can, for instance, delay her information reporting to a later period than her actual arrival time, so that she will face fewer competing drivers or only low-value drivers, when being assigned. By so doing the driver's

\footnotetext{
${ }^{3}$ Another approach is to assume that the parking manager has some a priori expectations of the future arrivals. See Parkes et al. (2003) as an example.
} 
payment will be reduced. It may also be possible that a driver deliberately submits a lower than her true value in order to be assigned at a later period where she pays less.

To illustrate this, let us consider a simple dynamic allocation of one parking slot with three demanding drivers (Table 3). At time $t=1$, drivers 1 and 2 are present, waiting to be assigned. Under the static assignment, driver 1 will be allocated a slot with payment of 80 , which is the highest reported value from unassigned drivers (in this case, only driver 2). But driver 1 could do better by lying about $e_{1}$ or $v_{1}$. If she instead reported that her arrival time $e_{1}=2$, then the slot will first be allocated to driver 2 with zero payment until $t=3$. Then after driver 2 leaves the slot driver 1 gets assigned with a payment at 60 . Alternatively, to reduce her payment driver 1 could lower her reported valuation to 79 while declaring her true arrival time. This would lead to the same allocation outcome: driver 2 is assigned the slot at $t=1$ with payment 79 (the highest reported value from unassigned drivers, and there is only driver 1 left unassigned at $t=1$ ) and driver 1 is assigned at time period 3 (after driver 1 leaves) with a payment of 60 . In both cases, driver 1 gets better off than submitting her true type information, as she is able to park with a lower payment. Therefore, truth telling is not a dominant strategy for driver 1 and she has the incentive to report untruthful information. To prevent this, we shall consider a different mechanism to perform the dynamic parking slot assignment.

Table 3: Illustrative example of untruthful reporting with implementation of the static assignment to dynamic parking

\begin{tabular}{|l|l|l|l|l|}
\hline Driver & Arrival time $e_{i}$ & Latest waiting $w_{i}$ & Departure time $d_{i}$ & Valuation $v_{i}$ \\
\hline 1 & 1 & 3 & 4 & 100 \\
\hline 2 & 1 & 2 & 3 & 80 \\
\hline 3 & 2 & 3 & 6 & 60 \\
\hline
\end{tabular}

To formally set up the dynamic parking slot assignment problem, let us consider $J$ slots in a public parking facility to accommodate demand from $I$ drivers over a finite horizon of $T$ periods. At the beginning of each time period $t=1, \ldots, T$, the parking manager updates information about the inventory of available parking slots. This can be realized by using a variety of existing technologies, such as ultrasonic sensors (Park et al., 2008) and wireless sensors (Panja et al., 2011). On the other hand, as long as truth telling is implementable, the parking inventory can also be updated with the user arrival and departure time information, and the outcome of the allocation thus far.

Because the parking manager has no a priori information about future arrivals, the parking slot assignment will be performed based on the available information at hand - unassigned drivers who have sent requests and vacant slots, in order to maximize total welfare of assigned drivers in each period. We assume that only after a driver arrives in the parking area will she be able to report her type information $\boldsymbol{\theta}_{i}$. This can be generalized to the case that the driver receives information submission request only when she is in close proximity to the parking facility, such that the in-vehicle device (or the driver's Smartphone apps) becomes active in communication with the parking manager. With this constraint, any reported arrival time $e_{i}^{\prime}$ is assumed to be no earlier than the driver's true arrival time $e_{i}$.

At the beginning of each time period $t$, the parking manager first updates the vacant slot inventory:

$$
J_{b}^{t}=J_{a}^{t-1}+n_{l}^{t}
$$

where $J_{b}^{t}$ is the number of available slots at the beginning of $t$, which equals the vacant slots at the end of the previous period $t-1, J_{a}^{t-1}$, plus those that are just released due to the departure of some parked vehicles, $n_{l}^{t}$. The subscript $b$ and $a$ denote before and after assignment respectively. After the parking inventory is updated, the parking manager looks at the pool of drivers who submitted their type information $\left(e_{i}, w_{i}, d_{i}, v_{i}\right)$ and are waiting for assignment. We use $I_{b}^{t}$ to denote the total number of such 
drivers at time $t$ prior to slot allocation. $I_{b}^{t}$ consists of two groups of drivers: those who are "leftovers" from the previous period, $I_{a}^{t-1}$, and those who just arrived at $t, m^{t}:{ }^{4}$

$$
I_{b}^{t}=I_{a}^{t-1}+m^{t}
$$

The proposed parking slot allocation mechanism, which we term $\boldsymbol{M}\left(\boldsymbol{a}^{t}, \boldsymbol{p}^{t}\right)$, consists of the following allocation and payment rules:

\section{Allocation rule}

At each time period $t=1, \ldots, T$, the parking manager assigns $\min \left(I_{b}^{t}, J_{b}^{t}\right)$ slots to the $I_{b}^{t}$ unassigned, requesting drivers. If there is a shortage of slot supply as opposed to the number of requesting drivers, then only the first $J_{b}^{t}$ drivers ordered by valuation will be given slots, which maximizes the total welfare of assigned drivers at $t$. Otherwise, there is no capacity shortage and every agent will be assigned a slot. The allocation at each $t$ is mathematically formalized as follows:

$$
\begin{aligned}
& \boldsymbol{a}^{t}=\operatorname{argmax}_{x^{t} \in X^{t} \sum_{i=1}^{I^{t}} v_{i}\left(\boldsymbol{x}^{t}\right) x_{i}^{t}} \\
& \text { s.t. } x_{i}^{t}=\left\{\begin{array}{l}
1 \text { if driver } i \text { is assigned to a slot at } t \\
0 \quad \text { otherwise }
\end{array} \quad \forall i \in\left\{1, \ldots, I_{b}^{t}\right\}\right. \\
& \sum_{i=1}^{I_{t}} x_{i}^{t} \leq \min \left(I_{b}^{t}, J_{b}^{t}\right)
\end{aligned}
$$

where $\boldsymbol{x}^{t}$ is a $0-1$ vector representing a possible allocation outcome at $t$. Each element $x_{i}^{t}$ indicates whether driver $i$ is assigned a slot. $\boldsymbol{X}^{\boldsymbol{t}}$ is the set of all possible allocation outcomes. $\boldsymbol{a}^{t}=\left(a_{1}^{t}, \ldots, a_{I_{b}^{t}}^{t}\right)$ gives the allocation outcome that maximizes total welfare.

Once a driver $i$ is assigned at a period, denoted by $t_{i}^{0}$, the driver will be out of the parking slot assignment process in the subsequent periods. Mechanism $\boldsymbol{M}\left(\boldsymbol{a}^{t}, \boldsymbol{p}^{t}\right)$ stipulates that an assigned driver starts using the parking slot and makes payment at the end of $w_{i}$. At first sight, it may seem less intuitive that the assigned driver $i$ has to waiting beyond $t_{i}^{0}$ even after they are assigned a slot at $t_{i}^{0}$. However, this requirement is necessary and important to prevent lying of drivers, as we will show below. It is conceivable that $\boldsymbol{M}\left(\boldsymbol{a}^{t}, \boldsymbol{p}^{\boldsymbol{t}}\right)$ may not be well received by drivers when they are told to be assigned a slot but still have to wait till their submitted latest waiting. To overcome this, practical implementation may skip releasing the assignment time to drivers when they are assigned, but instead inform drivers only when they can actually use the assigned parking slots.

Corresponding to the actual slot assignment time, the actual payment made by driver $i$, if assigned a slot, will also be made at $w_{i}$. Indeed, determination of drivers' payment under $\boldsymbol{M}\left(\boldsymbol{a}^{t}, \boldsymbol{p}^{t}\right)$ will require calculation of payment at each of the time periods from $t_{i}^{0}$ to $w_{i}$. We further assume that a driver will receive zero utility if: 1 ) a slot is assigned beyond her latest waiting $\left.w_{i} ; 2\right)$ the length of parking is shorter than her planned duration $d_{i}-w_{i}$. The former assumption is reasonable in that the driver would lose her maximum patience and already left the parking facility if the allocation were made beyond $w_{i}$. The latter assumption is justified on the ground that asking the driver to come back to get her car out of the parking facility earlier than her anticipated departure time will interrupt her planned activities in the trip. Translating them into reporting types, we only allow the reported latest waiting time $w_{i}^{\prime}$ and departure time $d_{i}^{\prime}$ to be no later and no earlier than the driver's respective truthful values $w_{i}$ and $\left.d_{i}: 1\right) w_{i}^{\prime} \leq w_{i} ; 2$ ) $d_{i}^{\prime} \geq d_{i}$. After the allocation decision is made, the parking inventory and the pool of unassigned drivers are updated:

\footnotetext{
${ }^{4}$ It is possible that not all arriving drivers will choose to stay upon arrival. If the parking manager makes available historic average waiting time information for assigned drivers, then those future-arriving drivers whose maximum waiting time (i.e., $w_{i}-e_{i}$ ) is much smaller than the historic average may decide not to choose the parking facility (i.e. submit type information and wait) in the first place.
} 
$J_{a}^{t}=J_{b}^{t}-\min \left(I_{b}^{t}, J_{b}^{t}\right)$

$I_{a}^{t}=I_{b}^{t}-\min \left(I_{b}^{t}, J_{b}^{t}\right)$

The above allocation is accompanied by the following payment rule in order to elicit truthful information reporting:

\section{Payment rule}

As is already shown, drivers may lie about their type if simply imposing the static case payment to drivers in the dynamic parking slot assignment. To ensure that the allocation mechanism $\boldsymbol{M}\left(\boldsymbol{a}^{\boldsymbol{t}}, \boldsymbol{p}^{\boldsymbol{t}}\right)$ is incentive compatible, an alternative payment rule needs to be devised. To this end, we first introduce the concepts of virtual and actual payments.

Definition 1. Without loss of generality, we suppose that driver $i$ with type $\boldsymbol{\theta}_{i}=\left(e_{i}, w_{i}, d_{i}, v_{i}\right)$ is allocated a slot at $t_{i}^{0}\left(e_{i} \leq t_{i}^{0} \leq w_{i}\right)$. Then virtual payment for driver $i$ is defined as follows:

1) At $t_{i}^{0}$, virtual payment is $p_{i, v}^{t_{i}^{0}}=\sum_{k \neq i} v_{k}\left(\boldsymbol{b}^{t_{i}^{0}}\right) b_{k}^{t_{i}^{0}}-\sum_{k \neq i} v_{k}\left(\boldsymbol{a}^{t_{i}^{0}}\right) a_{k}^{t_{i}^{0}}$, where $\boldsymbol{b}^{t_{i}^{0}}$ and $\boldsymbol{a}^{t_{i}^{0}}$ are, respectively, the allocation outcome by solving the same allocation problem (10.1)-(10.3) when driver $i$ is excluded/included at $t_{i}^{0}$. The virtual payment for driver $i$ is equal to the highest valuation among unallocated drivers, and is the same for all allocated drivers at $t_{i}^{0}$.

2) At each $t \in\left[t_{i}^{0}+1, w_{i}\right]$, virtual payment calculation is based on the following principle: we assume that driver $i$ is kept unallocated until $t$. Conditional on this, we first identify the lowest value among assigned drivers in each $t_{p} \in\left[e_{i}, t-1\right]$. That value is labeled $v_{-i_{v}, \min }^{t_{p}}$. If at $t_{p}$ no driver is allocated, then $v_{-i_{v}, \text { min }}^{t_{p}}=M$, where $M$ is a very big number. We then calculate the virtual payment for $i, p_{i, v}^{t}$. Provided that $i$ is allocated at $t$, we optimally allocate the remaining drivers to the available slots. The price each assigned driver pays is the valuation of the highest unassigned driver's, which we denote by $p_{-i_{v}, v}^{t}$. If $p_{-i_{v}, v}^{t}$ is no greater than any of the $v_{-i_{v}, \min }^{t_{p}}$ for $t_{p} \in\left[e_{i}, t-1\right]$, then virtual payment $p_{i, v}^{t}$ is equal to $p_{-i_{v}, v}^{t}$. Otherwise, virtual payment $p_{i, v}^{t}$ is equal to $M$. Mathematically, the virtual payment of $i$ at $t$ is:

$$
p_{i, v}^{t}=\left\{\begin{array}{cc}
p_{-i_{v}, v}^{t} & \text { if } p_{-i_{v}, v}^{t} \leq \min _{t_{p} \in\left[e_{i}, t-1\right]} v_{-i_{v}, \min }^{t_{p}} \\
M & \text { otherwise }
\end{array} \quad \forall t \in\left[t_{i}^{0}+1, w_{i}\right]\right.
$$

When $p_{i, v}^{t} \neq M$, then (13) calculates, for each $t$ between $t_{i}^{0}+1$ and $w_{i}$, the minimum possible valuation driver $i$ can have that enables her to obtain a slot at $t$ but not before $t$. If $p_{i, v}^{t}=M$, it implies that driver $i$ obtaining a slot at $t$ but not before $t$ is not possible. We add subscript $v$ to subscript $i$ in $v_{-i_{v}, \min }^{t_{p}}$ and $p_{-i_{v}, v}^{t}$, to reflect the fact that the corresponding allocations are virtual-driver $i$ is assumed only present and "virtually" allocated a slot at $t$. Note that we do not consider virtual allocation of driver $i$ during the time interval $\left[e_{i}, t_{i}^{0}-1\right]$, as in reality $i$ will stay unassigned until $t_{i}^{0}$.

From Definition 1 we can see that, once we know $t_{i}^{0}$, virtual payment in each period of $\left[t_{i}^{0}, w_{i}\right]$ is independent of $v_{i}$. Indeed, the calculation of virtual payment at $t_{i}^{0}$ can be unified with that for $\left[t_{i}^{0}+1, w_{i}\right]$. We present the independence feature as Lemma 1. As we shall see in sub-Section 3.2, independence is critical for eliciting truthful information reporting from drivers.

Lemma 1. In the payment rule under $\boldsymbol{M}\left(\boldsymbol{a}^{t}, \boldsymbol{p}^{t}\right)$, driver $i$ 's virtual payment for any given period in $\left[t_{i}^{0}, w_{i}\right]$ is independent of her reported valuation.

Proof. See Definition 1. 
The actual payment made by driver $i$, when assigned a slot, is the minimum of all virtual payments from $t_{i}^{0}$ to $w_{i}$, as defined below.

Definition 2. The actual payment of driver $i$ with type $\boldsymbol{\theta}_{i}=\left(e_{i}, w_{i}, d_{i}, v_{i}\right)$, provided that she is allocated a slot, is the minimum value of all virtual payments during $\left[t_{i}^{0}, w_{i}\right]$ :

$$
p_{i}^{a}=\left\{\begin{array}{lr}
\min _{t \in\left[t_{i}^{0}, w_{i}\right]} p_{i, v}^{t} & \text { if } t_{i}^{0} \leq w_{i} \\
0 & \text { if } i \text { is not allocated by the end of } w_{i}
\end{array}\right.
$$

The essence of Equation (14) is to identify the minimum possible valuation of driver $i$ such that the driver is allocated a slot, and that minimum possible valuation is the actual payment. Definitions 1 and 2 suggest that, as long as the driver is assigned a slot, actual payment is always no greater than the driver's reported valuation. We formalize this as Lemma 2 below.

Lemma 2. The payment rule under $\boldsymbol{M}\left(\boldsymbol{a}^{t}, \boldsymbol{p}^{t}\right)$ guarantees non-negative net utility received by drivers participating in the dynamic parking slot assignment.

Proof. For any driver $i$, if she is not assigned a slot, then no payment will be made and the driver will receive zero utility. If the driver is assigned a slot, then Equation (14) guarantees that the actual payment is no greater than the virtual payment at $t_{i}^{0}$. By Proposition 2, net utility received by driver $i$ would be non-negative if the actual payment were the virtual payment at $t_{i}^{0}$. Therefore, the net utility based on the payment rule (14) must give the assigned driver non-negative net utility.

It is worth mentioning that even if the driver is assigned a slot, her payment could still be zero, when there exists at least one zero virtual payment. This occurs when the number of parking slots is no fewer than the number of waiting drivers in the actual allocation at $t_{i}^{0}$, or in at least one virtual allocation in $\left[t_{i}^{0}+1, w_{i}\right]$. When the first zero virtual payment appears for an assigned driver, the parking manager will be ensured that the driver will not pay anything for using the slot, and consequently there is no need to keep the driver waiting until $w_{i}$. The driver shall be informed at this time point and start using the assigned slot. This adjustment is incorporated into $\boldsymbol{M}\left(\boldsymbol{a}^{\boldsymbol{t}}, \boldsymbol{p}^{\boldsymbol{t}}\right)$ in this study. The numerical analysis in Section 4 further investigates how much waiting time can be saved due to this adjustment.

We again use the example in Table 3 to demonstrate how virtual and actual payments are calculated under $\boldsymbol{M}\left(\boldsymbol{a}^{\boldsymbol{t}}, \boldsymbol{p}^{t}\right)$. Let us focus on driver 1. At period 1, drivers 1 and 2 submit their type information. Because driver 1 has a higher valuation, she will be assigned the slot, i.e., $t_{1}^{0}=1$. Driver 1 's virtual payment at $t_{1}^{0}, p_{1, v}^{1}$, is 80 . Virtual payment calculation then moves to period 2 . Because driver 1 could not be allocated at period 2 (no available slot), virtual payment of driver 1 at this period, $p_{1, v}^{2}$, is $M$. The calculation of virtual payment for driver 1 at period 3 is more complicated. First, we exclude driver 1 from the waiting driver list for periods 1 and 2. Driver 2 will be first allocated the slot at period 1, and will start using the slot from the beginning of period 2 till the beginning of period 3. Then driver 2 leaves and the slot becomes vacant. Driver 1 will then be placed to the slot. The highest valuation among unassigned drivers (only driver 3 in this case), $p_{-1_{v}, v}^{3}$, is 60 . Note that we need to compare $p_{-1_{v}, v}^{3}$ with $v_{-1_{v}, \min }^{1}$ and $v_{-1_{v}, \min }^{2}$. It can be easily seen that $v_{-1_{v}, \min }^{1}=80$ and $v_{-1_{v}, \min }^{2}=M$. Since $p_{-1_{v}, v}^{3}=60<$ $\min \left(v_{-1_{v}, \min }^{1}, v_{-1_{v}, \min }^{2}\right)=\min (80, M)=80$, virtual payment at period $3 p_{1, v}^{3}=p_{-1_{v}, v}^{3}=60$. The actual payment for driver $1, p_{1}^{a}$, is equal to $\min \left(p_{1, v}^{1}, p_{1, v}^{2}, p_{1, v}^{3}\right)=\min (80, M, 60)=60$.

\subsection{Truthfulness of the dynamic parking slot allocation mechanism}

With the exposition of the allocation and payment rules, we now turn to proving that $\boldsymbol{M}\left(\boldsymbol{a}^{t}, \boldsymbol{p}^{t}\right)$ is incentive compatible. The proof takes advantage of the monotonicity property of the proposed allocation rule, which has been discussed in the general online mechanism literature (e.g., Parkes, 2007). To begin, let us first introduce the definition of monotonic allocation. 
Definition 3. For any two drivers with types $\boldsymbol{\theta}_{1}=\left(e_{1}, w_{1}, d, v_{1}\right)$ and $\boldsymbol{\theta}_{2}=\left(e_{2}, w_{2}, d, v_{2}\right)$, driver 1 arrives earlier, has a later latest waiting time, and has a higher valuation than driver 2 , i.e., $e_{1} \leq e_{2}$, $w_{1} \geq w_{2}$, and $v_{1} \geq v_{2}$. The monotonic allocation rule states that, if driver 2 is assigned a slot, then driver 1 must also be assigned a slot if driver 1 replaces driver 2 in the allocation.

It is not difficult to see that the allocation rule of $\boldsymbol{M}\left(\boldsymbol{a}^{\boldsymbol{t}}, \boldsymbol{p}^{\boldsymbol{t}}\right)$ is monotonic. If driver 2 is allocated a slot at $t_{2}^{0} \in\left[e_{2}, w_{2}\right]$, then at the very least driver 1 will be assigned a slot at the same time ceteris paribus. If driver 1 has a sufficiently larger valuation or gets involved in the allocation process significantly earlier than driver 2 , it is possible that driver 1 will receive a parking place earlier than $t_{2}^{0}$. Note that the monotonicity rule governs not only the valuation but also the arrival and latest waiting times in a driver's type information. However, departure time is not involved. As shown later, truthfulness proof of a driver's departure time reporting is independent of the proof of the other three parameters.

The proof of truthful information reporting is composed of three parts: 1) truth telling of the arrivallatest waiting interval $\left.\left[e_{1}, w_{1}\right] ; 2\right)$ of the valuation $v_{i}$; and 3) of the departure time $d_{i}$. We present proofs for 1)-3) below in order. In proving each part of the truthfulness, the implicit assumption is that all other drivers' reporting is held constant. We first show that, under $\boldsymbol{M}\left(\boldsymbol{a}^{\boldsymbol{t}}, \boldsymbol{p}^{\boldsymbol{t}}\right)$, declaring the true arrival-latest waiting interval is the dominant strategy for any driver.

Proposition 4. For any driver $i$, the utility received is non-increasing by reporting a different arrivallatest waiting interval other than her true interval $\left[e_{i}, w_{i}\right]$ under $\boldsymbol{M}\left(\boldsymbol{a}^{\boldsymbol{t}}, \boldsymbol{p}^{\boldsymbol{t}}\right)$.

Proof. See Appendix A.

We now proceed with the truthfulness of the valuation $v_{i}$.

Proposition 5. For any driver $i$, reporting a different valuation other than $v_{i}$ cannot improve the driver's utility under $\boldsymbol{M}\left(\boldsymbol{a}^{t}, \boldsymbol{p}^{t}\right)$.

Proof. See Appendix B.

Finally, the truthfulness of $d_{i}$ is presented in Proposition 6.

Proposition 6. For any driver $i$, reporting a different than her true departure time does not give her additional utility under $\boldsymbol{M}\left(\boldsymbol{a}^{\boldsymbol{t}}, \boldsymbol{p}^{\boldsymbol{t}}\right)$.

Proof. For the departure time $d_{i}$, recall that a driver will receive zero utility if allocated a slot for a period that ends earlier than her true departure time $d_{i}$. In practice, heavy penalty should be implemented if a driver stays longer than her declared parking time. Therefore, the driver will not report a departure time earlier than $d_{i}$. On the other hand, if the driver reports a longer than her true length of parking, i.e., $d_{i}^{\prime} \geq d_{i}$, her net utility remains constant if she ends up leaving the parking facility at $d_{i}$. In addition, staying longer than the one's planned duration at the parking place does not increase her utility, but will make the driver miss other scheduled activities. Therefore, the driver has no incentive to report a longer than her true departure time either.

Propositions 4-6 completes the proof of the truthfulness of driver information reporting.

Proposition 7. The dynamic parking slot allocation mechanism $\boldsymbol{M}\left(\boldsymbol{a}^{t}, \boldsymbol{p}^{\boldsymbol{t}}\right)$ induces truth telling of drivers. In other words, for any driver $i$, she cannot be better off by not declaring her true type information $\boldsymbol{\theta}_{i}$.

Proof. Use Propositions 4, 5, and 6.

\subsection{Further discussions on the dynamic parking mechanism}

In addition to eliciting truthful information reporting, similar to the static case we are interested in 1) whether $\boldsymbol{M}\left(\boldsymbol{a}^{t}, \boldsymbol{p}^{t}\right)$ always offers drivers non-negative utility; and 2) whether the payment is guaranteed to be non-negative. The first question is already answered by Lemma 2. Definition 2 further suggests that 
payment made by an assigned driver is always non-negative. (Of course, payment will be zero if not allocated.) Thus non-negative payment also holds under $\boldsymbol{M}\left(\boldsymbol{a}^{t}, \boldsymbol{p}^{t}\right)$.

Because the times when a driver submits her type information and when she actually starts using the assigned slots are in general different, the valuation of parking may decrease between the two time points. Up to now value discounting is not explicitly dealt with in $\boldsymbol{M}\left(\boldsymbol{a}^{\boldsymbol{t}}, \boldsymbol{p}^{\boldsymbol{t}}\right)$, which may be appropriate if: 1) discounting is really small and can be reasonably neglected; or 2) when reporting type, a given driver $i$ is asked specifically to submit their valuation of parking at time $w_{i}$. The latter case implicitly incorporates the value discounting effect (from when the driver submits her type information to when she starts using the assigned slot). It can also be justified on the ground that, at any time point, the parking manager makes the slot allocation decision based on the actual utility drivers will receive when using the assigned slots in the future. One might think of an alternative mechanism that requires each driver to report her current valuation (i.e., valuation at the time of reporting) and her discount factor separately, while decision making is still based on the actual utility received by drivers. However, truth telling cannot be guaranteed because monotonicity of the allocation will be violated. For a given discount factor, reporting a later $w_{i}$ also suggests a lower valuation at $w_{i}$ due to further discounting. It therefore becomes indeterminate as to whether having a later $w_{i}$ will increase the chance of being assigned a slot.

Recall that under $\boldsymbol{M}\left(\boldsymbol{a}^{t}, \boldsymbol{p}^{t}\right)$ a driver may be able to use the assigned parking slot earlier than $w_{i}$, if zero virtual payment is encountered prior to $w_{i}$. This will result in extra benefits to those drivers due to reduction in waiting and consequently value discounting, which is not anticipated and quantified by the parking manager when making assignment decisions, because at the time of assignment the parking manager does not have a priori knowledge about future driver arrivals.

It is often customary to examine when total welfare obtained under a dynamic mechanism has a lower bound (sometimes referred to as the competitive ratio) with respect to the value from the corresponding offline, static mechanism (Parkes, 2007). Such a lower bound, however, does not exist for $\boldsymbol{M}\left(\boldsymbol{a}^{\boldsymbol{t}}, \boldsymbol{p}^{\boldsymbol{t}}\right)$. The intuition is that if a large number of lower-value drivers arrive at the parking facility earlier enough than their higher-value fellow drivers, such that all slots are occupied by the low-value drivers. In addition, the low-value drivers plan to stay longer than the high-value drivers would. So by the time the unassigned high-value drivers have to leave, no vacant slots are available. With an offline parking slot assignment mechanism, slots will be first assigned to those high-value drivers. The ratio of welfare gains under the dynamic and static mechanisms will continuously decrease with increasing discrepancy of valuation between the high- and low-value drivers.

A special case of the dynamic parking slot assignment is morning commuter parking, where ample parking slots exist at the beginning of the day. Commuting drivers continuously arrive at the parking facility during the morning peak hours, and each desires one parking slot for the rest of the day. Therefore, the pool of vacant parking slots is non-increasing throughout the morning peak. On the other hand, as long as excess supply of parking slots is present, payment will be zero, and persist until when the number of demanding drivers is greater than the number of empty slots. Only part of the demand can be accommodated and non-zero payment for the assigned drivers will result. Afterwards, the parking facility will stop accepting new arriving vehicles. The drivers who are left unassigned have to leave and search for available parking at other parking facilities.

So far we have assumed that payment is not an explicit function of time. However, in real world parking management it may be that the mechanism-specific payment is designed based on the duration of parking. To this end, we also consider an extension of $\boldsymbol{M}\left(\boldsymbol{a}^{t}, \boldsymbol{p}^{t}\right)$, termed $\boldsymbol{M}^{\prime}\left(\boldsymbol{a}^{t}, \boldsymbol{p}^{t}\right)$, which asks drivers to submit their valuation information on a per unit time basis. Accordingly, total payment is calculated by multiplying the unit time payment by the reported length of parking $\left(d_{i}-w_{i}\right)$. Before presenting the formal proof of whether this new mechanism induces truth telling, we use a simple example to illustrate the difference in allocation outcome between lump sum (as in $\boldsymbol{M}\left(\boldsymbol{a}^{\boldsymbol{t}}, \boldsymbol{p}^{\boldsymbol{t}}\right)$ ) and per unit time valuation reporting. 
Consider three drivers who demand the use of two parking slots have the same actual arrival time $\left(e_{i}=1, i=1,2,3\right)$. Their types $\boldsymbol{\theta}_{i}$ differ in the latest waiting $w_{i}$, departure time $d_{i}$, and the valuation of parking slots $v_{i, 0}$, as shown in Table 4 below. The numbers in parentheses in the last column are the corresponding total valuation. ${ }^{5}$

Table 4: Drivers' type information

\begin{tabular}{|l|l|l|l|l|}
\hline Driver & $\begin{array}{l}\text { Arrival } \\
\text { time } e_{i}\end{array}$ & $\begin{array}{l}\text { Latest waiting } \\
w_{i}\end{array}$ & $\begin{array}{l}\text { Departure } \\
\text { time } d_{i}\end{array}$ & $\begin{array}{l}\text { Value per unit time } v_{i, 0} \\
\text { (total value in parenthesis) }\end{array}$ \\
\hline 1 & 1 & 2 & 12 & $30(300)$ \\
\hline 2 & 1 & 2 & 5 & $35(105)$ \\
\hline 3 & 1 & 3 & 9 & $20(120)$ \\
\hline
\end{tabular}

For now let us suppose that all reported information is truthful. Under $\boldsymbol{M}\left(\boldsymbol{a}^{\boldsymbol{t}}, \boldsymbol{p}^{\boldsymbol{t}}\right)$, drivers 1 and 3 will be assigned slots at $t=1$. Because drivers 1 and 3 will stay beyond driver 2's latest waiting $w_{2}$, driver 2 will leave the parking facility unassigned, although she has the highest unit time valuation. As a consequence, $\boldsymbol{M}\left(\boldsymbol{a}^{t}, \boldsymbol{p}^{\boldsymbol{t}}\right)$ could be unjust for drivers like driver 2 who have higher unit time valuation of parking slots but need to park for shorter periods of time.

The allocation rule under $\boldsymbol{M}^{\prime}\left(\boldsymbol{a}^{t}, \boldsymbol{p}^{t}\right)$, which asks drivers to submit their valuation on a unit time basis, is to maximize the sum of unit valuations of assigned drivers in each period. If a driver $i$ is allocated at $t_{i}^{0}$, her virtual payment at $t_{i}^{0}$ will be the product of the highest unit time valuation among the unassigned drivers and the reported length of stay $\left(d_{i}-w_{i}\right)$. Similar modification will be made to virtual payments in $\left(t_{i}^{0}+1, w_{i}\right]$. Specifically, virtual payment at any $t \in\left[t_{i}^{0}+1, w_{i}\right]$ would be:

$$
p_{i, v}^{t}=\left\{\begin{array}{cc}
p_{-i_{v}, v, 0}^{t}\left(d_{i}-w_{i}\right) & \text { if } p_{-i_{v}, v, 0}^{t} \leq \min _{t_{p} \in\left[e_{i}, t-1\right]} v_{-i_{v}, \min , 0}^{t_{p}} \\
M & \text { otherwise }
\end{array} \quad \forall t \in\left[t_{i}^{0}+1, w_{i}\right]\right.
$$

where $p_{-i_{v}, v, 0}^{t}$ is the highest unit time value among unassigned drivers at $t$ (with $i$ guaranteed a slot); $v_{-i_{v}, \min , 0}^{t_{p}}$ the lowest unit time value among assigned drivers at $t_{p}$ (with $i$ excluded from the assignment). The actual payment for $i$ is still the minimum of all virtual payments. Table 5 shows the actual payments for drivers in Example 1. Under $\boldsymbol{M}\left(\boldsymbol{a}^{t}, \boldsymbol{p}^{t}\right)$, payment for the allocated drivers 1 and 3 is the same, equal to driver 2's total valuation, 105. When $\boldsymbol{M}\left(\boldsymbol{a}^{\boldsymbol{t}}, \boldsymbol{p}^{\boldsymbol{t}}\right)$ is replaced by $\boldsymbol{M}^{\prime}\left(\boldsymbol{a}^{\boldsymbol{t}}, \boldsymbol{p}^{\boldsymbol{t}}\right)$, at period 1 drivers 1 and 2 will be assigned slots, with virtual payment equal to the unit value of driver 3 times the reported length of stay of drivers 1 and 2, respectively: $p_{1, v}^{1}=20 \times(12-2)=200$ and $p_{2, v}^{1}=20 \times(5-2)=60$. At period 2, driver 1 will not be allocated in the virtual allocation, because drivers 2 and 3 would be assigned in period 1 and they occupy the parking slots beyond $w_{1}$. Therefore $p_{1, v}^{2}=M$. The actual payment for driver 1 is $p_{1}^{a}=\min \left(p_{1, v}^{1}, p_{1, v}^{2}\right)=\min (200, M)=200$. Similarly, $p_{2, v}^{2}=M$; the actual payment for driver 2 is $p_{2}^{a}=\min \left(p_{2, v}^{1}, p_{2, v}^{2}\right)=\min (60, M)=60$. As a result, the parking manager will collect more revenue under $\boldsymbol{M}^{\prime}\left(\boldsymbol{a}^{t}, \boldsymbol{p}^{t}\right)$ (Table 5).

Table 5: Actual driver payment under $\boldsymbol{M}\left(\boldsymbol{a}^{\boldsymbol{t}}, \boldsymbol{p}^{\boldsymbol{t}}\right)$ and $\boldsymbol{M}^{\prime}\left(\boldsymbol{a}^{\boldsymbol{t}}, \boldsymbol{p}^{\boldsymbol{t}}\right)$

\begin{tabular}{|l|l|l|}
\hline Driver & Payment under $\boldsymbol{M}\left(\boldsymbol{a}^{\boldsymbol{t}}, \boldsymbol{p}^{\boldsymbol{t}}\right)$ & Payment under $\boldsymbol{M}^{\prime}\left(\boldsymbol{a}^{\boldsymbol{t}}, \boldsymbol{p}^{\boldsymbol{t}}\right)$ \\
\hline 1 & 105 & $200(=20 *(12-2))$ \\
\hline 2 & 0 (unassigned) & $60(=20 *(5-2))$ \\
\hline
\end{tabular}

\footnotetext{
${ }^{5} 300$ comes from 30 times 10 , which is $12-2\left(d_{i}-e_{i}\right)$.
} 


\begin{tabular}{|l|l|l|}
3 & 105 & 0 (unassigned) \\
\hline Total & 210 & 260 \\
\hline
\end{tabular}

Proposition 8. Mechanism $\boldsymbol{M}^{\prime}\left(\boldsymbol{a}^{t}, \boldsymbol{p}^{t}\right)$ which implements unit time valuation reporting induces truth telling among drivers.

Proof: The proof resembles that for Proposition 7. The monotonicity of the allocation rule, which now is based on the unit time valuation, remains valid. It is clear that truthfully reporting $\left[e_{i}, w_{i}\right]$ still maximizes the chance of driver $i$ being allocated, everything else being equal. The length of parking does not affect the truthfulness of a driver's valuation reporting $v_{i, 0}$ : if reporting $v_{i, 0}$ is better than reporting any untrue unit time valuation, then multiplying $v_{i, 0}$ by $\left(d_{i}-w_{i}\right)$ does not change the driver's value reporting behavior. Proof of truthfulness about $d_{i}$ is also the same as for Proposition 6. Because now payment is a function of the length of parking, i.e., $d_{i}-w_{i}$, which is basically a multiplier of the payment in a unit time period, drivers are further disincentivized to report earlier than latest waiting and later than the actual departure time.

While both $\boldsymbol{M}\left(\boldsymbol{a}^{t}, \boldsymbol{p}^{t}\right)$ and $\boldsymbol{M}^{\prime}\left(\boldsymbol{a}^{t}, \boldsymbol{p}^{t}\right)$ are incentive compatible and implement parking slot assignment in a myopic way, $\boldsymbol{M}^{\prime}\left(\boldsymbol{a}^{\boldsymbol{t}}, \boldsymbol{p}^{\boldsymbol{t}}\right)$ is even more myopic in that allocation is made based on valuation of the current period. This increases the chance of being assigned a slot for drivers who have a high value of time but demand relatively short use of parking. Consequently, there can be more slots made available in subsequent periods after those high-value-of-time drivers leave the parking facility. From the perspective of parking slot utilization efficiency, $\boldsymbol{M}^{\prime}\left(\boldsymbol{a}^{\boldsymbol{t}}, \boldsymbol{p}^{\boldsymbol{t}}\right)$ could be welfare improving compared to $\boldsymbol{M}\left(\boldsymbol{a}^{\boldsymbol{t}}, \boldsymbol{p}^{\boldsymbol{t}}\right)$. On the other hand, $\boldsymbol{M}^{\prime}\left(\boldsymbol{a}^{t}, \boldsymbol{p}^{t}\right)$ focuses really just on welfare that occurs at the present time, therefore lacking a long-term view of the true utility drivers can derive from parking. We will investigate, through the ensuing numerical analysis, which of the two effects plays a more important role.

\section{Numerical analysis}

\subsection{Setup}

This section implements the dynamic mechanims proposed in the previous section for a hypothetical parking assignment process. We consider 200 drivers arriving and leaving over a time horizon of 20 periods. For a given driver $i$, her arrival time $e_{i}$ is generated from a discrete uniform distribution between 1 and 20. We also consider drivers' arrival time to follow Poisson distributions, with results reported in Appendix C. Given $e_{i}$, the latest waiting time of the driver, $w_{i}$, is randomly drawn from another discrete uniform distribution between the generated $e_{i}$ and 20. Assuming that a driver will park for at least one period once starting using the assigned slot, the departure time $d_{i}$ is drawn from a discrete uniform distribution between $w_{i}+1$ and $21{ }^{6}$ At the end of the planning horizon all occupying drivers have to vacate their slots. The valuation of each driver is drawn from the discrete uniform distribution between 10 and 40. The total number of parking slots is 100. All slots are empty at the beginning of the time horizon.

In what follows, a Monte Carlo simulation approach is adopted to gain robust insights about $\boldsymbol{M}\left(\boldsymbol{a}^{\boldsymbol{t}}, \boldsymbol{p}^{\boldsymbol{t}}\right)$ and $\boldsymbol{M}^{\prime}\left(\boldsymbol{a}^{t}, \boldsymbol{p}^{t}\right)$. Under each meachnism, 50 simulation runs are performed. We choose box plots to present most of the results. ${ }^{7}$

\footnotetext{
${ }^{6} \mathrm{We}$ set the latest departure time to be 21 while the arrival is limited to 20 to ensure that any randomly generated driver can park for at least one time period.

${ }^{7}$ In each of the subsequent box plots, the two boundaries of a box denote the 25 th and 75 th percentile values; the red line the median. The whiskers extend to the most extreme data points that are not considered outliers, and outliers are plotted as red crosses individually. The maximum whisker length (between the whisker and the closer box boundary) is 1.5 times the length of the box.
} 


\subsection{Results with $M\left(a^{t}, p^{t}\right)$}

This sub-section presents the results from simulating $\boldsymbol{M}\left(\boldsymbol{a}^{t}, \boldsymbol{p}^{t}\right)$. The ratio of allocated drivers in total drivers by time of arrival is shown in Figure 1. The medians are equal to one for the first 10 periods, when sufficient capacity is present. Then capacity becomes constrained: only a portion of the arriving drivers can be allocated starting from the 11th period. The ratio decreases as the available slots become scarcer. However, with more drivers depart from the parking facility after the 13th period, a greater portion of newly arriving drivers will obtain slots, as manifested by the gradual increase of the ratios. At the $19^{\text {th }}$ and $20^{\text {th }}$ periods, the medians of the ratio return to one as the number of available slots exceeds the number of waiting drivers at these periods.

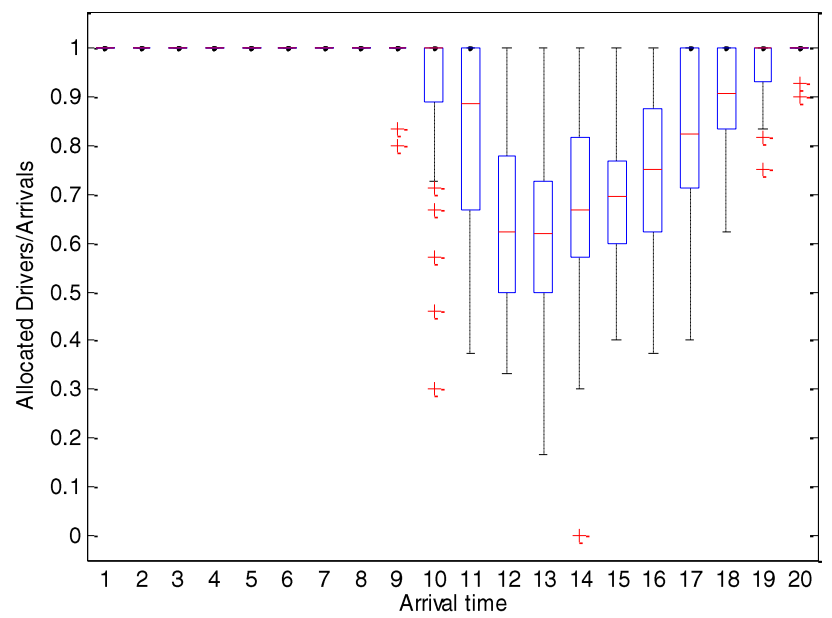

Figure 1: Ratio of allocated drivers in total drivers by time of arrival

Figure 2 shows the number of drivers that are assigned a slot (panel (a)) and that actually start using a slot (panel (b)) in each period. In panel (a), the median of the number of assigned drivers is roughly constant from the first to the ninth period, during which sufficient slot supply persists. The median is around 10, which is consistent with the expected number of arrivals in each period $(200 \times 0.05=10)$. Starting from the 10th period slot constraints become apparent, explaining the drop in the number of assigned drivers. After the 13th period, the number of assigned drivers starts increasing, because of more departures as shown in Figure 1. Near the end of the time horizon, much more slots become vacant, resulting in an even higher number of assigned drivers (which encompass both waiting drivers who arrived in earlier periods and new arrivals) than in the beginning.

Panel (b) shows the number of drivers who actually start using the assigned slots (at their respective $w_{i}$ 's or at the time when they first encounter a zero virtual payment). Drivers who receive zero virtual payment at the time of allocation (i.e., $t_{i}^{0}$ ) do not need to wait to use the slot. This explains the same number of drivers in panels (a) and (b) for the first nine periods. Once parking demand exceeds slot supply, virtual payment becomes positive and the assigned drivers have to wait till $w_{i}$, or when a zero virtual payment appears. 


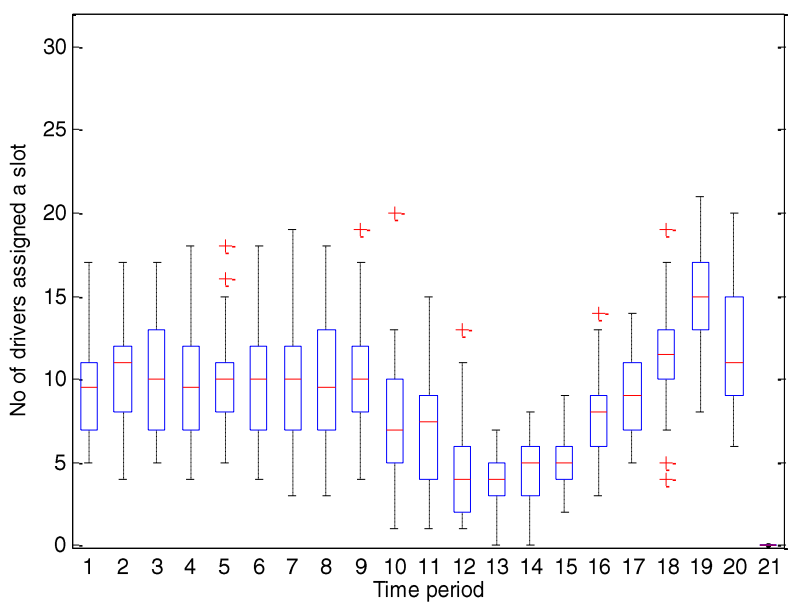

(a)

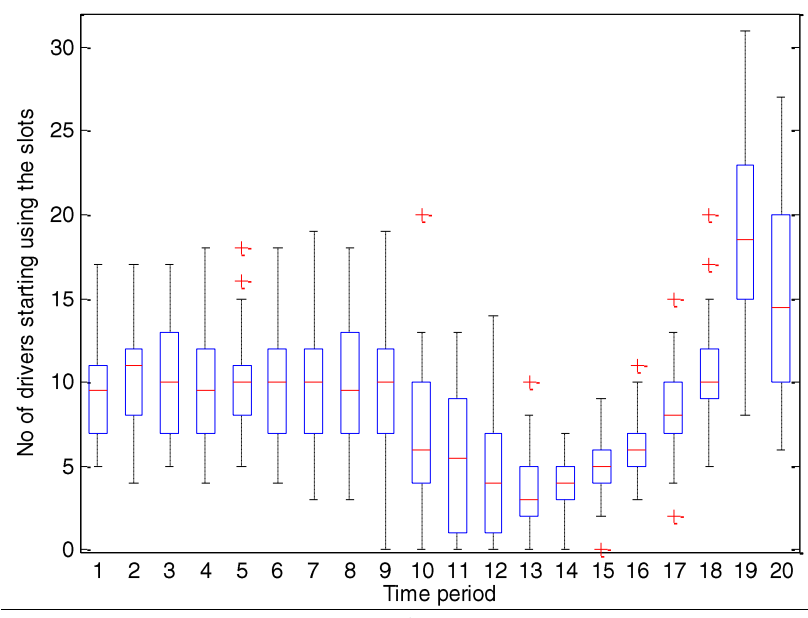

(b)

Figure 2: Number of drivers who are assigned a slot (a) and start using the assigned slot (b) in each period

Figure 3 presents the average waiting time prior to allocation (i.e., $t_{i}^{0}-e_{i}$ ) among assigned drivers, plotted against the time of arrival. In the first half of the time horizon, drivers do not have to wait to be assigned after arrival because there is ample supply of parking slots. For the other half, where parking supply encounters a shortage, non-zero pre-assignment waiting will occur. As more drivers leave the parking facility near the end of the time horizon, more slots become available to waiting drivers, which help reduce the average pre-assignment waiting for new arrivals.

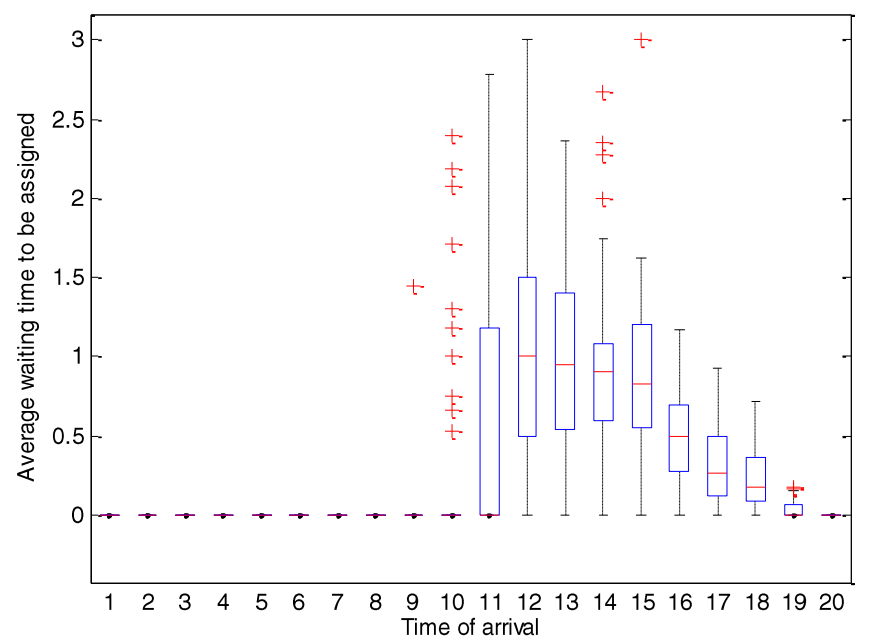

Figure 3: Average pre-assignment waiting time for drivers against drivers' time of arrival

Figure 4 plots the average post-assignment waiting time among assigned drivers, by time of arrival. Under $\boldsymbol{M}\left(\boldsymbol{a}^{t}, \boldsymbol{p}^{t}\right)$ the average post-assignment waiting time is always less than one period. For the first half of the time horizon, where excess capacity persists, drivers are assigned slots upon their arrival, and virtual payment at the arrival time is zero (due to excess capacity). Drivers will start using the assigned slots right away, with no payment. Comparing the post assignment time to pre-assignment time in Figure 3 further reveals that the average time for post-assignment waiting is almost always shorter than for preassignment waiting. 


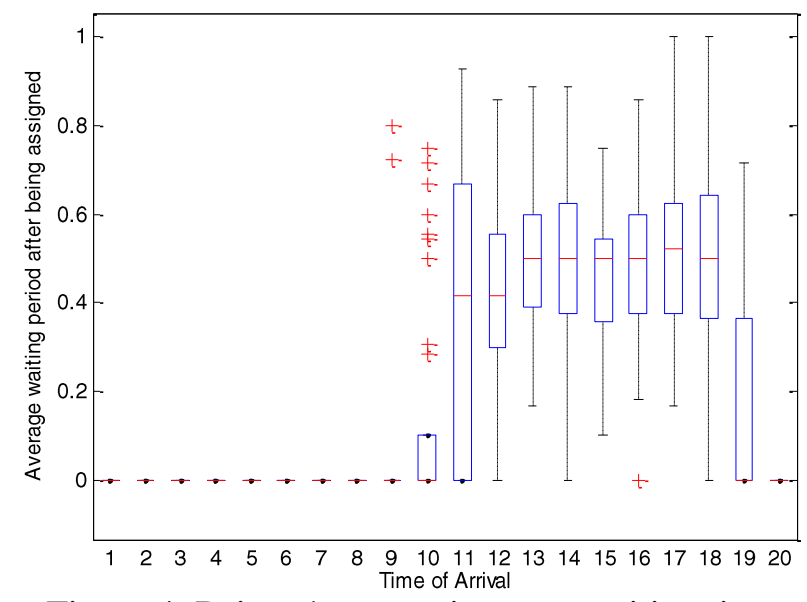

Figure 4: Drivers' post-assignment waiting time

Besides waiting time, drivers' actual payment is also important. Figure 5 presents the average payment per assigned driver as a function of: drivers' arrival time $\left(e_{i}\right)$ and valuation $\left(v_{i}\right)$. In panel (a), the actual payments are zero in the first half of the time horizon when excess parking supply exists. Drivers start to pay for using slots when capacity constraints appear. With greater shortage of slots, high-value drivers are more likely to be left unassigned, which contributes to increase in payment. Near the end of the time horizon, more slots become available because of more departures; this mitigates the demand-supply imbalance and consequently reduces payment. In particular, ample capacity would most likely re-appear at period 20, leading to zero payment for new arriving drivers.

When the average payment is plotted against drivers' valuation, panel (b) shows two general trends: 1) the average (median) payment increases with valuation; 2) the dispersion of payment is more significant with higher valuation. These trends are not difficult to interpret: higher valuation allows more pricing room. If a driver really values parking, then she will be assigned with higher priority despite greater price to pay. As long as the price is below her valuation, it would be acceptable. On the other hand, higher valuation increases the chance of assignment at an earlier period, which can reduce the actual payment as the time interval for virtual payment calculation $\left[t_{i}^{0}, w_{i}\right]$ becomes longer. Wider scattering of payment then results due to the two effects.

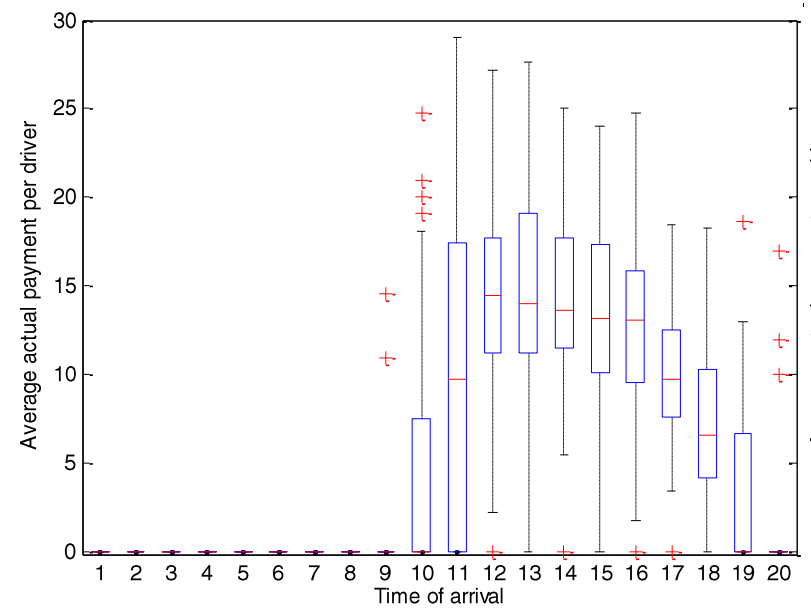

(a)

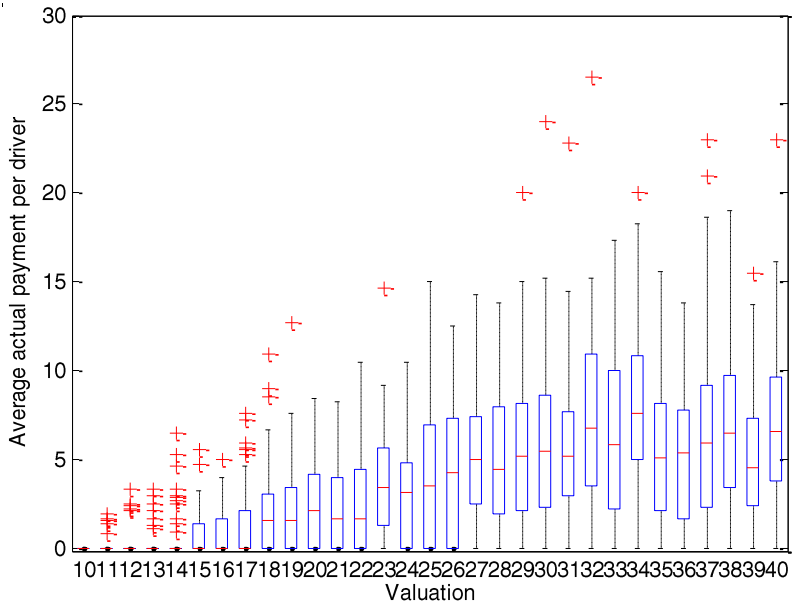

(b)

Figure 5: Average payment per assigned driver with respect to: a) time of arrival; b) valuation

We sum up the payments and welfare gains across drivers by their time of assignment $\left(t_{i}^{0}\right)$ for each simulation run, calculate the ratio of total payment over total welfare gains for each period, and then take 
the average over 50 simulation runs. The averaged ratio over periods is presented in Figure 6. Overall, the payment-welfare ratio is always less than 0.6 , with the peak reached during the 13th-16th periods. During the earlier parts of the time horizon no payment occurs; the ratio is zero. More vacant slots appear in the ending periods, which reduce payment. The last bar in Figure 6 gives the average payment-welfare ratio at the system level. Overall, about $20 \%$ of welfare will be deprived from drivers in order to induce truthful information reporting under $\boldsymbol{M}\left(\boldsymbol{a}^{t}, \boldsymbol{p}^{t}\right)$.

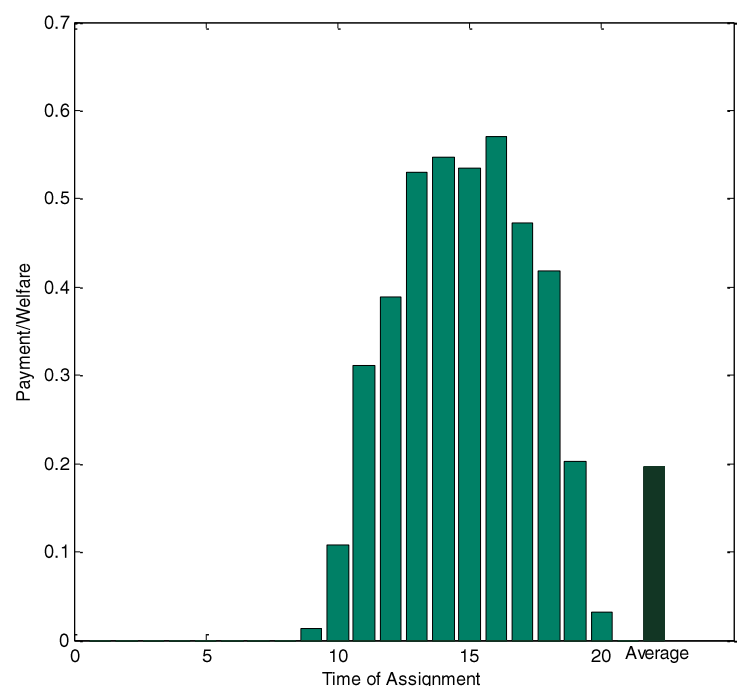

Figure 6: Ratio of total payment over welfare gained by time of assignment, averaged over 50 runs

We further investigate the effect of $\boldsymbol{M}\left(\boldsymbol{a}^{t}, \boldsymbol{p}^{t}\right)$ on welfare distribution over different driver groups. In Figure 7 we divide drivers into six valuation groups and present two metrics: total payment-welfare ratio among assigned drivers and the percentage of assigned drivers. For each group, the metric values are the average over 50 runs. From panel (a), we observe that higher valuation is associated with larger paymentvaluation ratios (except for the last valuation group 35-40), which is consistent with the fact that highervalue drivers can afford to pay more (and disproportionately so) in order to be assigned. Panel (b) suggests that higher-value drivers are more likely to receive slots: all drivers in the highest valuation group will get a parking slot; in contrast the portion will reduce to $70 \%$ for drivers in the lowest valuation group.

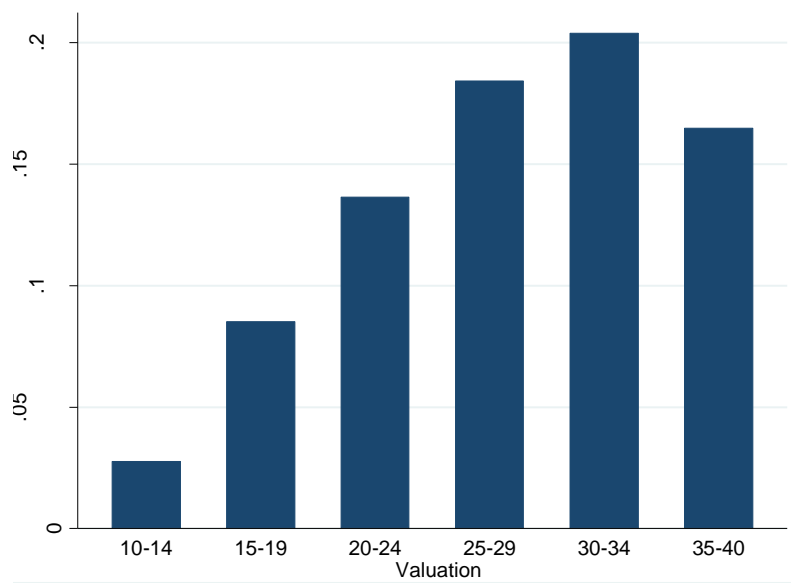

(a)

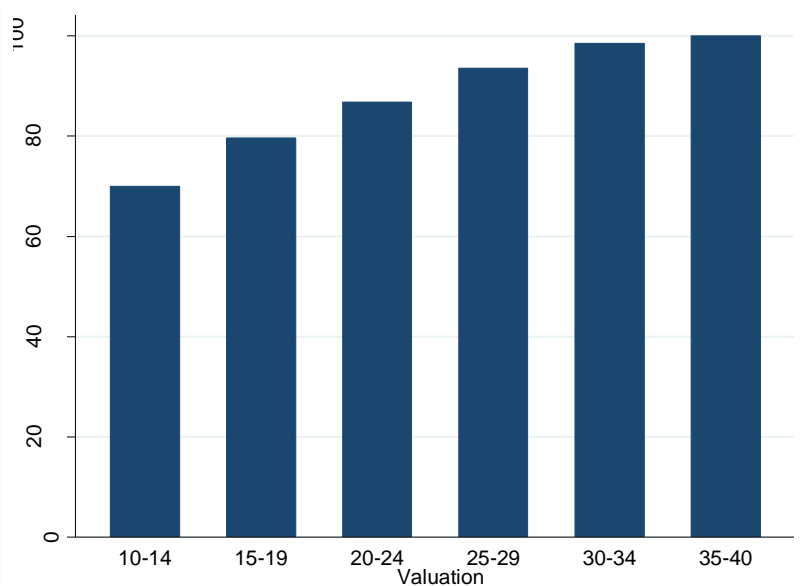

(b) 
Figure 7: Ratio of payment/valuation and percentage of assigned drivers by valuation group

\subsection{Comparing $M\left(a^{t}, p^{t}\right)$ with $M^{\prime}\left(a^{t}, p^{t}\right)$}

We have shown in sub-Section 3.3 that $\boldsymbol{M}^{\prime}\left(\boldsymbol{a}^{\boldsymbol{t}}, \boldsymbol{p}^{\boldsymbol{t}}\right)$, a variant of $\boldsymbol{M}\left(\boldsymbol{a}^{\boldsymbol{t}}, \boldsymbol{p}^{\boldsymbol{t}}\right)$ under which drivers report unit time valuation, is also incentive compatible. Using the same generated drivers, we compare the allocation outcomes from implementing $\boldsymbol{M}\left(\boldsymbol{a}^{\boldsymbol{t}}, \boldsymbol{p}^{\boldsymbol{t}}\right)$ (lump sum valuation) and $\boldsymbol{M}^{\prime}\left(\boldsymbol{a}^{\boldsymbol{t}}, \boldsymbol{p}^{\boldsymbol{t}}\right)$ (per unit time valuation). The distribution of total welfare, average payment per assigned driver, the number of drivers assigned, and total payment over 50 simulation runs are presented in Figure 8 (a)-(d). We observe lower medians for all four metrics under $\boldsymbol{M}^{\prime}\left(\boldsymbol{a}^{t}, \boldsymbol{p}^{t}\right)$.

As mentioned in sub-Section 3.3, the difference in total welfare between $\boldsymbol{M}\left(\boldsymbol{a}^{\boldsymbol{t}}, \boldsymbol{p}^{\boldsymbol{t}}\right)$ and $\boldsymbol{M}^{\prime}\left(\boldsymbol{a}^{\boldsymbol{t}}, \boldsymbol{p}^{\boldsymbol{t}}\right)$ is driven by two opposing forces: on the one hand, unit time valuation allows higher unit-time-value drivers to gain priority in the allocation. If the parking time of those drivers is relatively short, then more slots could be made available to other drivers in latter periods, thereby increasing total welfare. On the other hand, the objective of allocation under $\boldsymbol{M}^{\prime}\left(\boldsymbol{a}^{\boldsymbol{t}}, \boldsymbol{p}^{\boldsymbol{t}}\right)$ is to maximize total valuation of assigned drivers only at the current period, which is not aligned with the drivers' true overall valuation of parking. This inconsistency leads to inferior welfare gains under $\boldsymbol{M}^{\prime}\left(\boldsymbol{a}^{\boldsymbol{t}}, \boldsymbol{p}^{\boldsymbol{t}}\right)$. Our results suggest that the latter force is dominant, as shown in panel (a) of Figure 8.

Under $\boldsymbol{M}^{\prime}\left(\boldsymbol{a}^{\boldsymbol{t}}, \boldsymbol{p}^{\boldsymbol{t}}\right)$, drivers with higher unit time value and shorter stay are preferred to drivers with lower unit time value and longer stay. Because payment is based on the product of the unassigned drivers' lower unit time value and the assigned drivers' shorter duration of stay, a lower average payment would result, as shown in panel (b). Although under $\boldsymbol{M}^{\prime}\left(\boldsymbol{a}^{t}, \boldsymbol{p}^{t}\right)$ each slot may be made available more frequently when preference is given to higher unit-time-value drivers whose stay is short, that particular type of drivers only accounts for a portion of the population. Panel (c) shows that the number of drivers that can be allocated with $\boldsymbol{M}^{\prime}\left(\boldsymbol{a}^{t}, \boldsymbol{p}^{t}\right)$ would be indeed slightly lower than with $\boldsymbol{M}\left(\boldsymbol{a}^{\boldsymbol{t}}, \boldsymbol{p}^{\boldsymbol{t}}\right)$. Due to the considerably lower average payment and the slightly smaller number of assigned drivers, total payment under $\boldsymbol{M}^{\prime}\left(\boldsymbol{a}^{t}, \boldsymbol{p}^{t}\right)$ will also be substantially lower than under $\boldsymbol{M}\left(\boldsymbol{a}^{t}, \boldsymbol{p}^{t}\right)$ (panel (d)). While individual assigned drivers may favor $\boldsymbol{M}^{\prime}\left(\boldsymbol{a}^{t}, \boldsymbol{p}^{t}\right)$ given the lower amount to pay for one's own parking, from the revenue generating perspective, the parking manager will strongly prefer $\boldsymbol{M}\left(\boldsymbol{a}^{t}, \boldsymbol{p}^{t}\right)$.

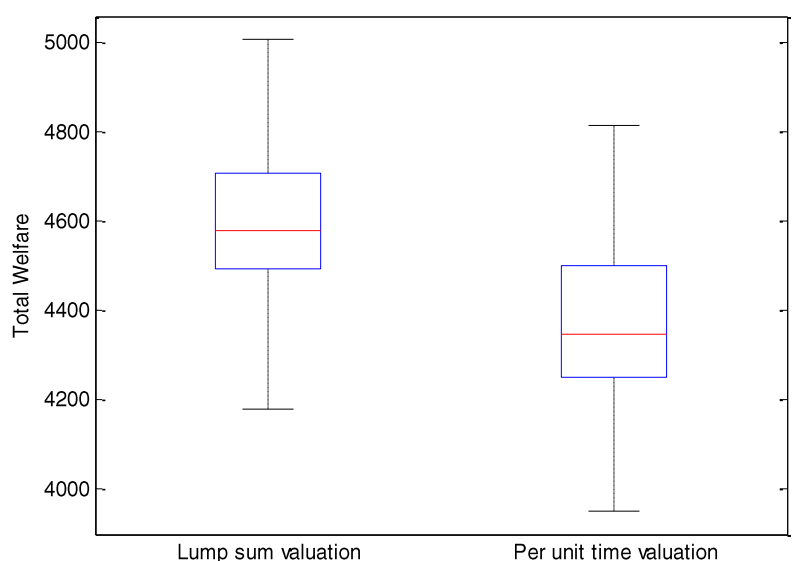

(a)

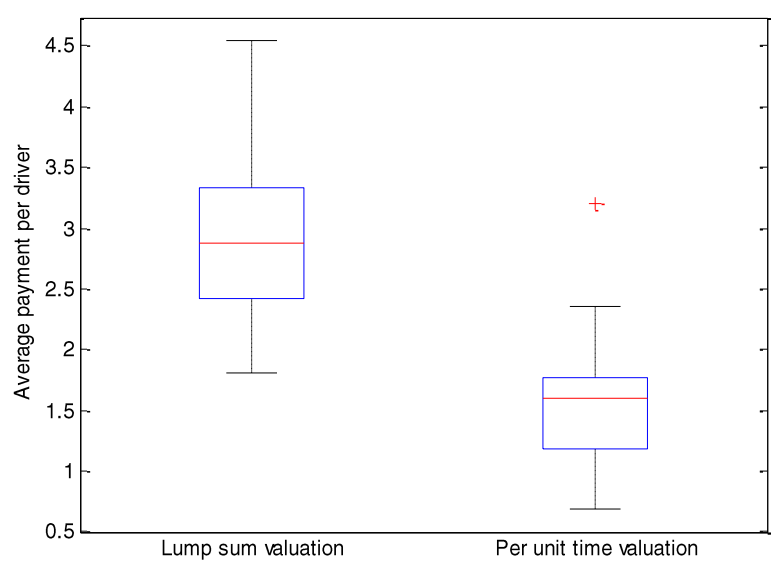

(b) 


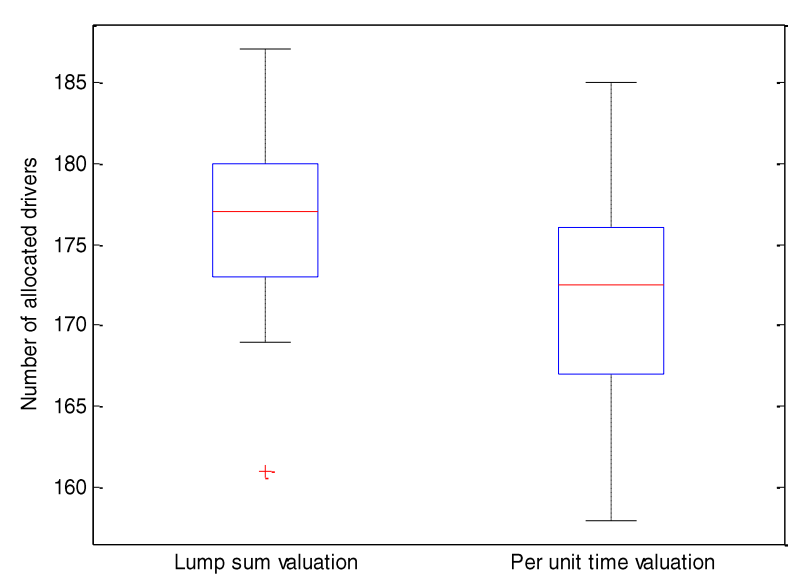

(c)

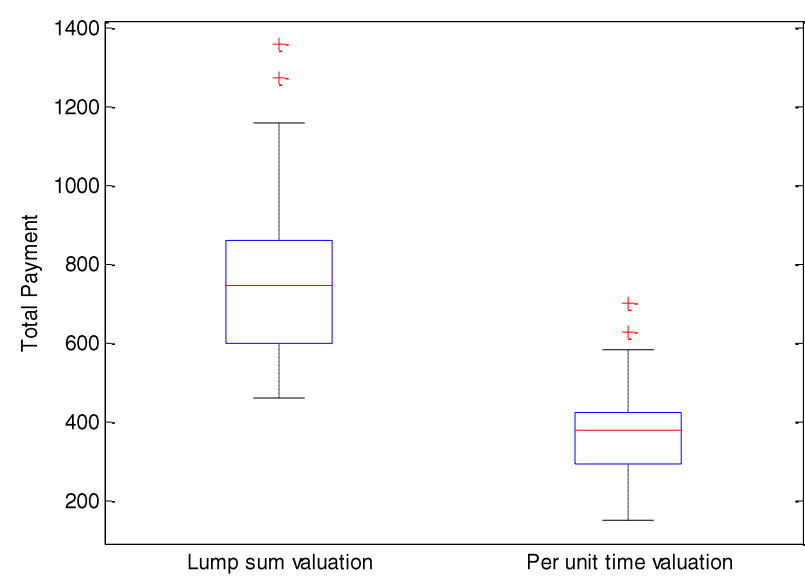

(d)

Figure 8: Comparison of allocation outcomes under mechanism with lump sum and per unit time based valuation reporting

\subsection{Comparing $M\left(a^{t}, p^{t}\right)$ with the state-of-the-practice and full information optimum}

We compare the allocation outcome from $\boldsymbol{M}\left(\boldsymbol{a}^{t}, \boldsymbol{p}^{t}\right)$ with the state-of-the-practice (SOTP) and the full information optimum. Under SOTP, drivers do not have to report type information. Allocation is based on a first-come-first-serve basis, and mechanism-specific payment is irrelevant. When abundant parking slot supply is present, there will be no difference between $\boldsymbol{M}\left(\boldsymbol{a}^{t}, \boldsymbol{p}^{t}\right)$ and SOTP as everyone gets a slot. When there are more waiting drivers than available slots, waiting drivers will be randomly picked to fill the slots. Under the full information optimum, all drivers' arrival, waiting and departure times are known to the parking manager in advance and the allocation is made at one shot. Figure 9 demonstrates the distributions of total welfare among assigned drivers with 50 simulation runs. On average, $\boldsymbol{M}\left(\boldsymbol{a}^{\boldsymbol{t}}, \boldsymbol{p}^{\boldsymbol{t}}\right)$ yields $\$ 237$ more total welfare (or $\$ 1.05 /$ park) than SOTP, but $\$ 116$ (or $\$ 0.65 /$ park) less than the full information optimum.

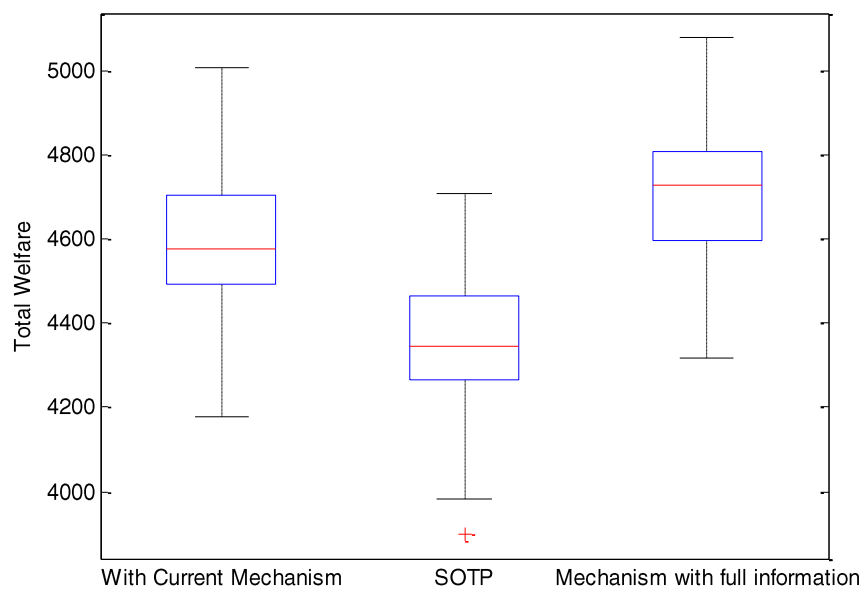

Figure 9: Comparison of total welfare under $\boldsymbol{M}\left(\boldsymbol{a}^{t}, \boldsymbol{p}^{t}\right)$,SOTP, and the full information optimum

\subsection{Sensitivity of length of time period interval}

Finally, we perform sensitivity analysis with respect to the length of a unit period. We assume that the total number of drivers remains 200 and consider five choices of total periods: 5, 10, 20, 40, and 60. The 
larger the total periods, the shorter the length of one unit period and the smaller the average number of arrivals per period. Results on total payment and welfare are shown in Figure 11, which are again averages over 50 simulation runs.

One may expect that assignment with longer unit time period yields larger overall social welfare as the parking manager will have greater information about driver arrivals when performing assignment. The results, however, show that the reverse is true. This is because longer unit period leads to lower utilization of the parking slots (our results show that a lower portion of drivers ends up being assigned parking slots when the length of a period becomes small). The average payment per assigned driver is generally also higher when the length of a period is larger, plausibly because more drivers now will be waiting to be assigned in each period. Figure 12 further demonstrates the average value over 50 simulation runs of average payment per assigned driver by time of arrival, when 5, 10, 40, and 60 periods are considered. From both the shape of the pricing curves and the standard deviation statistics, it is evident that the change in price is more gradual with more periods, thereby increasing its acceptability by drivers. Therefore, as long as it is operationally feasible, more frequent assignments (i.e., smaller length of a unit period) would be desirable from both social welfare and individual driver perspectives.

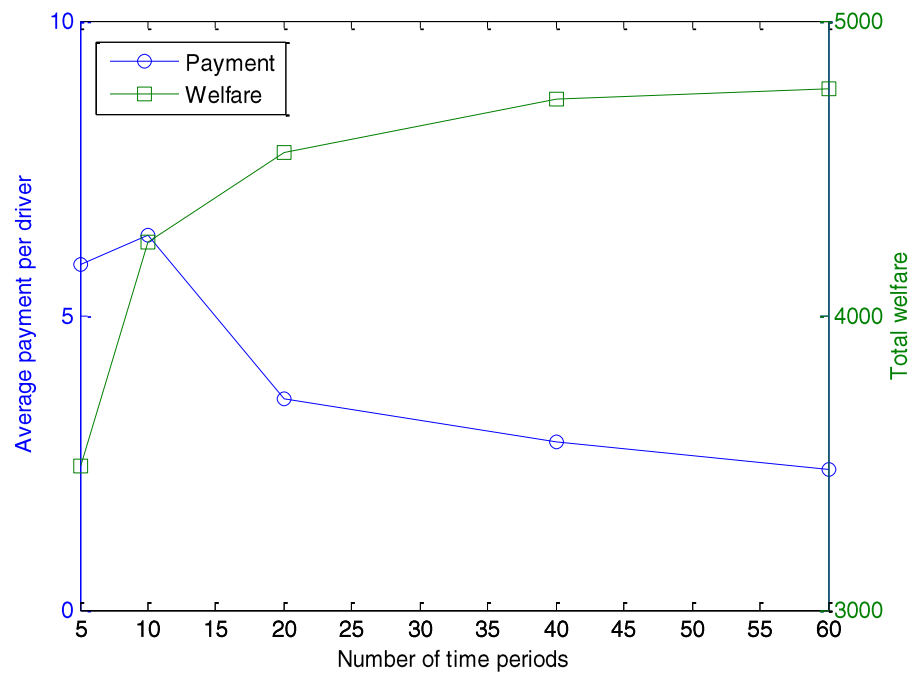

Figure 11: Sensitivity of average payment and total welfare to the number of time periods

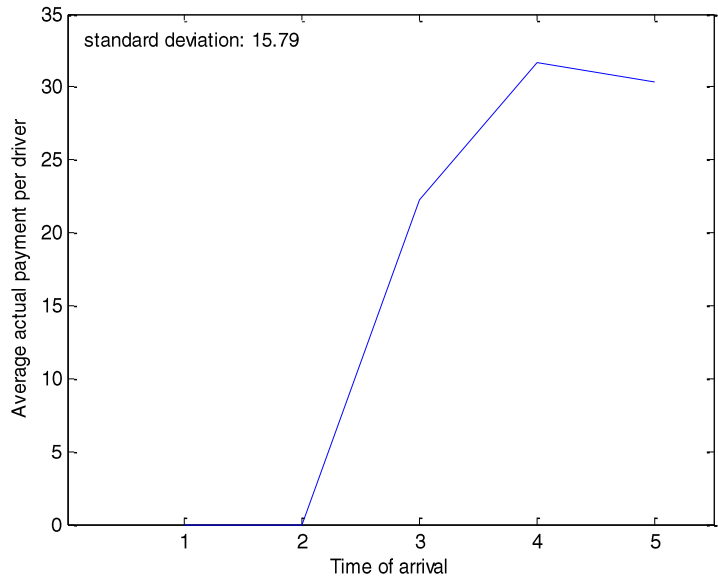

(a)

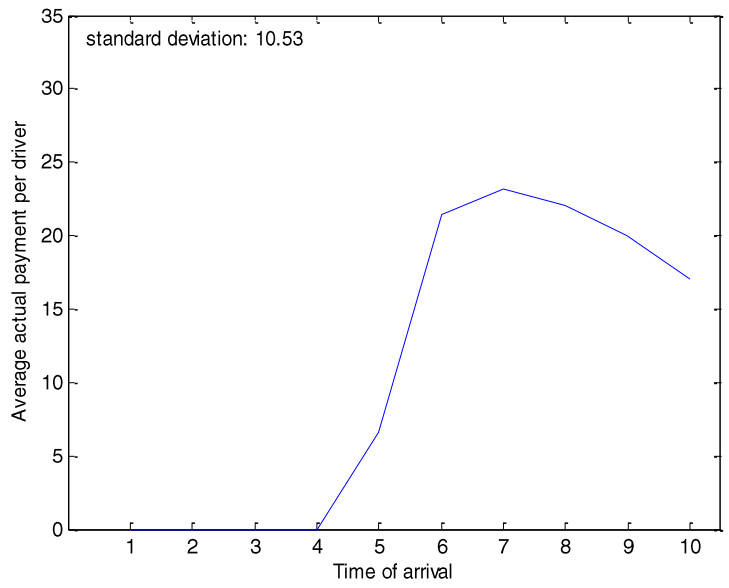

(b) 


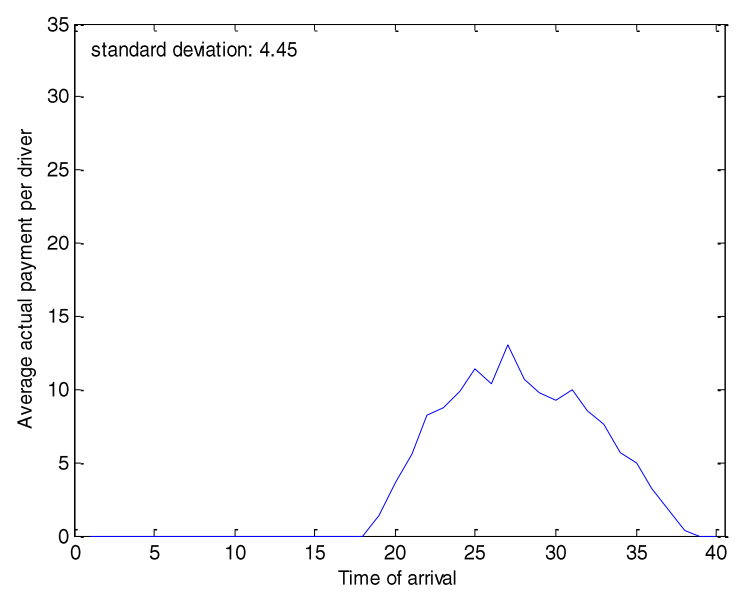

(c)

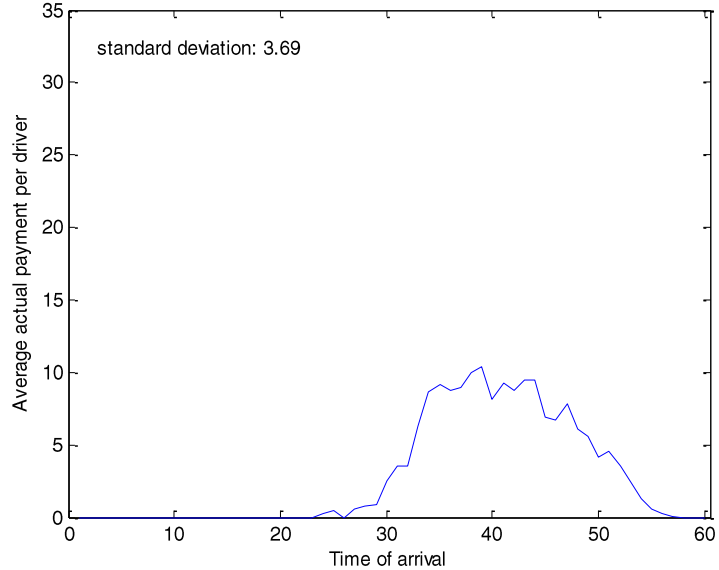

(d)

Figure 12: Sensitivity of average payment variation to the number of time periods

\section{Conclusion}

This study investigated agent-based parking slot assignment enabled by recent advances in sensing, infrastructure-to-vehicle, and vehicle-to-infrastructure communication technologies to improve social welfare. An important part in the assignment process is information collection from drivers by the parking manager. The parking manager needs to guard against drivers' strategically misreporting private information, which would deviate the allocation outcome from social optimum. To this end, this paper introduced mechanism design principles to allocating parking slots to heterogeneous demanding drivers.

We started with static allocation, where drivers value the same slot differently and any two slots can be valued differently by the same driver. We showed that welfare-maximizing allocation coupled with payment equivalent to a driver's externality induces truth telling and achieves the social optimum allocation outcome. The investigation was then extended to dynamic parking slot assignment, where each driver is assumed to report arrival, latest waiting, and departure times in addition to valuation. Assuming that the parking manager does not have information about future arrivals, a myopic optimal allocation rule and a new price scheme were put forward to elicit truthful information reporting from drivers. A variant of the dynamic mechanism when valuation was reported on a per-unit-time basis was further considered, for which we showed that drivers' truth telling holds as well. Under the proposed mechanisms, each assigned driver pays a non-negative amount to the parking manager, and drivers' net utility is always nonnegative. Extensive numerical experiments were performed to gain further insights into implementation of the dynamic mechanisms.

For future research, we recommend a few directions. First, this paper does not explicitly consider what drivers would do if unassigned, which is particularly relevant to low-value drivers. Learning that there is small chance of getting a parking slot, low-value drivers may end up not considering the parking facility. Given the public nature of the parking management, addressing the equity issue is important for practical implementation of the mechanisms. Second, in urban areas competition among multiple parking facilities is not uncommon. Consequently a driver may submit parking requests to more than one facility to maximize her chance of parking. To model the parking manager-driver interactions, a game theoretic framework may be further coupled with the mechanisms proposed in the present study. Third, the mechanisms introduced here belong to the category of direct mechanisms, in which drivers are asked to report their complete private information. Although direct mechanisms are more intuitive, privacy concerns may arise. Indirect mechanisms that avoid full revealing of private information could be an alternative to achieve the same allocation outcome. Fourth, uncertainties are often involved in parking, for 
example, the time to depart from the occupied parking slot (e.g., if one needs to wait for a doctor). Future studies may explicitly model how drivers with different characteristics (e.g., risk averse vs. risk seeking) would report their valuation which would be an explicit function of parking time. Lastly, the present study focuses on public parking ownership. It would be interesting to explore private parking management which may start introducing informed parking mechanisms in conjunction with revenue management.

\section{Acknowledgement}

This research was funded in part by the National Science Foundation grants IIS-1213013, CCF1216096, DGE-0549489, IIP-1315169. Financial supports from the US Department of Transportation National University Rail Center (NURail) and Illinois Department of Transportation (METSI) are also gratefully acknowledged. The authors would like to thank Professor Richard Arnott and one anonymous reviewer for very constructive comments which helped us prepare an improved version of the paper.

\section{References}

1. Arnott, R., Palma, A. D., Lindsey, R., 1991. A temporal and spatial equilibrium analysis of commuter parking. Journal of public Economics 45(3), 301-335.

2. Ayala, D., Wolfson, O., Xu, B., Dasgupta, B., Lin, J., 2011. Parking slot assignment games. Proceedings of the 19th ACM SIGSPATIAL International Conference on Advances in Geographic Information Systems.

3. Ayala, D., Wolfson, O., Xu, B., DasGupta, B., Lin, J., 2012. Pricing of parking for congestion reduction. Proceedings of the 20th International Conference on Advances in Geographic Information Systems.

4. Berenger Vianna, M. M., Portugal, L. D. S., Balassiano, R., 2004. Intelligent transportation systems and parking management: implementation potential in a Brazilian city. Cities 21(2), 137-148.

5. Caicedo, F., 2010. Real-time parking information management to reduce search time, vehicle displacement and emissions. Transportation Research Part D: Transport and Environment 15(4), 228234.

6. Glazer, A., Niskanen, E., 1992. Parking fees and congestion. Regional Science and Urban Economics 22(1), 123-132.

7. Kokolaki, E., Karaliopoulos, M., Stavrakakis, I., 2011. Value of information exposed: wireless networking solutions to the parking search problem. Proceedings of the 8th International Conference on Wireless On-Demand Network Systems and Services (WONS).

8. Litman, T., 2011. Parking management comprehensive implementation guide. Technical report, Victoria Transport Policy Institute.

9. Mathur, S., Jin, T., Kasturirangan, N., Chandrasekaran, J., Xue, W., Gruteser, M., Trappe, W., 2010. ParkNet: drive-by sensing of road-side parking statistics. Proceedings of the 8th international conference on Mobile systems, applications, and services.

10. Nisan, N., 2007. Introduction to mechanism design. In Algorithmic Game Theory. Cambridge University Press, pp. 209-242.

11. Panja, B., Schneider, B., and Meharia, P., 2011. Wirelessly sensing open parking spaces: accounting and management of parking facility. Proceedings of AMCIS.

12. Park, W. J., Kim, B. S., Seo, D. E., Kim, D. S., and Lee, K. H., 2008. Parking space detection using ultrasonic sensor in parking assistance system. Proceedings of the IEEE Intelligent Vehicles Symposium.

13. Parkes, D. C., 2007. Online mechanisms. In Algorithmic Game Theory. Cambridge University Press, pp. 411-439. 
14. Parkes, D. C., and Singh, S. P., 2003. An MDP-Based Approach to Online Mechanism Design. In Advances in Neural Information Processing Systems (NIPS).

15. Qian, Z. S., Rajagopal, R., 2013. Optimal parking pricing in general networks with provision of occupancy information. Procedia-Social and Behavioral Sciences 80 (20th International Symposium on Transportation and Traffic Theory (ISTTT 2013)), 779-805.

16. Qian, Z. S., Rajagopal, R., 2014. Optimal occupancy-driven parking pricing under demand uncertainties and traveler heterogeneity: A stochastic control approach. Transportation Research Part B: Methodological 67, 144-165.

17. Shoup, D., 2005. Parking cash out, Report 532. Technical report, Planning Advisory Service, American Planning Association.

18. Shoup, D., 2006. Cruising for parking. Transport Policy 13(6), 479-486.

19. Thompson, R. G., Richardson, A. J., 1998. A parking search model. Transportation Research Part A: Policy and Practice 32(3), 159-170.

20. Verhoef, E., Nijkamp, P., Rietveld, P., 1995. The economics of regulatory parking policies: the (im) possibilities of parking policies in traffic regulation. Transportation Research Part A: Policy and Practice 29(2), 141-156.

21. Verroios, V., Efstathiou, V., Delis, A., 2011. Reaching available public parking spaces in urban environments using ad hoc networking. Proceedings of 12th IEEE International Conference on Mobile Data Management (MDM).

22. Wang, J.Y., Yang, H., Lindsey, R., 2004. Locating and pricing park-and-ride facilities in a linear monocentric city with deterministic mode choice. Transportation Research Part B 38(8), 709-731.

23. Wolfson, O., Xu, B., 2004. Opportunistic dissemination of spatio-temporal resource information in mobile peer to peer networks. Proceedings of the 15th International Workshop on Database and Expert Systems Applications. 


\section{Appendix A: Proof of Proposition 4.}

Suppose that driver $i$ has true type $\boldsymbol{\theta}_{i}=\left(e_{i}, w_{i}, d_{i}, v_{i}\right)$. Now driver $i$ reports instead $\boldsymbol{\theta}_{i}{ }^{\prime}=$ $\left(e_{i}{ }^{\prime}, w_{i}{ }^{\prime}, d_{i}, v_{i}\right)$. Recall that a driver has only limited misreporting about arrival time and latest waiting time: $e_{i}{ }^{\prime} \geq e_{i}$ and $w_{i}{ }^{\prime} \leq w_{i}$. Three allocation outcomes are possible with untruthful reporting:

1) Driver $i$ is not allocated with either $\left[e_{i}, w_{i}\right]$ or $\left[e_{i}{ }^{\prime}, w_{i}{ }^{\prime}\right]$. Evidently there is no payment and the driver receives zero utility in both cases.

2) Driver $i$ is assigned a slot with both $\left[e_{i}, w_{i}\right]$ and $\left[e_{i}{ }^{\prime}, w_{i}{ }^{\prime}\right]$. Suppose that the actual payment of driver $i$ when reporting $\left[e_{i}{ }^{\prime}, w_{i}{ }^{\prime}\right]$ is $p_{\left.i_{\left[i^{\prime}, w^{\prime}\right]}\right]}^{a}$, which is the lowest possible valuation $i$ could report to obtain a slot. When driver $i$ reports $\left[e_{i}, w_{i}\right]$, having $p_{i_{\left[i_{i}^{\prime}, w^{\prime}\right]}^{a}}^{a}$ still guarantees her a slot. The allocation could occur either

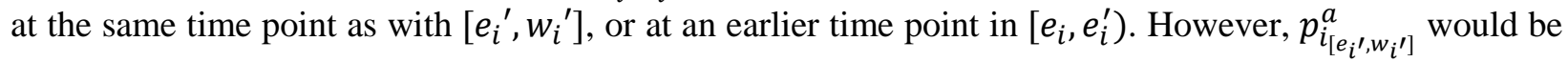
only an upper bound of the driver's minimum valuation when reporting $\left[e_{i}, w_{i}\right]$. Driver $i$ may be allocated a slot at interval $\left[e_{i}, e_{i}{ }^{\prime}\right)$ or $\left(w_{i}^{\prime}, w_{i}\right]$ with an even lower valuation. Therefore, the actual payment of driver $i$ by reporting $\left[e_{i}, w_{i}\right]$ is no greater than the actual payment with $\left[e_{i}{ }^{\prime}, w_{i}{ }^{\prime}\right]$.

3) Driver $i$ is assigned a slot when reporting $\left[e_{i}, w_{i}\right]$, but would end up being unallocated if reporting a tighter interval $\left[e_{i}{ }^{\prime}, w_{i}{ }^{\prime}\right]$. The utility received by the driver by misreporting, which would be zero, is not utility improving compared to the utility when declaring the truth.

Summing up, truth telling of $\left[e_{i}, w_{i}\right]$ is a dominant strategy for the driver.

\section{Appendix B: Proof of Proposition 5.}

We demonstrate the truthfulness of valuation reporting by case analysis:

1) Driver $i$ is not allocated a parking slot when reporting $v_{i}$. The highest valuation among unassigned drivers in all $t \in\left[e_{i}, w_{i}\right]$ must be no less than $v_{i}$. Driver $i$ may have done two things:

1a) $i$ may have reported a false valuation $v_{i}^{\prime}>v_{i}$, which results in the driver being assigned a slot at $t_{i \prime}^{0}$. The consequence must be that some other driver, who would have been assigned at $t_{i \prime}^{0}$, is "kicked out" and left unassigned at $t_{i \prime}^{0}$. The virtual payment at $t_{i}^{0}$ is the valuation of the "kicked-out" driver, because that driver has the highest valuation among unassigned drivers, and is no less than $v_{i}$. If $i$ also gets assigned in the virtual allocation at some of the subsequent periods in $\left[t_{i^{\prime}}^{0}+1, w_{i}\right]$, with virtual payment less than $M$, then by similar logic the virtual payment at such period(s) will be no less than $v_{i}{ }^{8}$ Therefore, the actual payment of driver $i$, which is the minimum of virtual payments in $\left[t_{i^{\prime}}^{0}, w_{i}\right]$, is no less than $v_{i}$. Reporting a higher than one's true valuation always gives a non-positive net utility if assigned.

1b) $i$ may have reported a false valuation $v_{i}^{\prime}<v_{i}$. By monotonicity of the allocation rule, driver $i$ will remain unassigned, i.e., no improvement in the driver's utility.

2) Driver $i$ is allocated a parking slot when reporting $v_{i}$. In this case driver $i$ may have done two things:

2a) $i$ may have reported a false valuation $v_{i}^{\prime}>v_{i}$. By monotonicity of the allocation rule, $i$ will remain assigned. Suppose that the time of allocation for $i$ is $t_{i \prime \prime}^{0}$, which may be identical to or earlier than $t_{i}^{0}$ $\left(t_{i \prime \prime}^{0} \leq t_{i}^{0}\right)$. Below we consider two scenarios:

i. There is no change in the period (termed $t_{i, \text { minpay }}$ ) from which the minimum of virtual payments is chosen as the actual payment in (14). Then by Lemma 2 virtual payment at $t_{i, \text { minpay }}$ can only be the maximum valuation among the unassigned drivers (and cannot

\footnotetext{
${ }^{8}$ Of course, if virtual payment is $M$, the statement still holds, as $M \gg v_{i}$.
} 
be $M)$. In addition, Lemma 1 indicates that the virtual payment at $t_{i, \text { minpay }}$ is identical whether driver $i$ reports $v_{i}^{\prime}$ or $v_{i}$. Consequently the actual payment remains unchanged.

ii. The period from which the minimum of virtual payments is chosen as the actual payment in (14) is different. It can only be that the driver is allocated at an earlier period $t_{i \prime \prime}^{0}<t_{i}^{0}$. When $t_{i \prime \prime}^{0}<t_{i}^{0}$ the payment of driver $i$ at $t_{i \prime \prime}^{0}$ is no less than $v_{i}$ as some other driver with valuation greater than $v_{i}$ has to be kicked out at $t_{i \prime \prime}^{0}$. For all the time periods $\left[t_{i \prime \prime}^{0}+1, t_{i}^{0}-1\right]$ the virtual payment for driver $i$ would be no lesser than $v_{i}$. However, by Lemma 1, virtual payments from $t_{i}^{0}$ to $w_{i}$ when reporting $v_{i}^{\prime}$ will be the same as when $i$ reports $v_{i}$, and we know at least at $t_{i}^{0}$ the virtual payment is no greater than $v_{i}$. It then becomes clear that the actual payment, which is the minimum of all virtual payments, will not be affected by the virtual payments prior to $t_{i}^{0}$ when $i$ reports $v_{i}^{\prime}$, and remains the same as when $i$ reports $v_{i}$.

Overall, the driver has no incentive to report a higher valuation.

2b) $i$ may have reported a false valuation $v_{i}^{\prime}<v_{i}$. If $v_{i}^{\prime}$ is sufficiently low such that $i$ becomes unassigned, evidently this is not utility-improving. If $v_{i}^{\prime}$ is lower than $v_{i}$ and keeps $i$ receiving a parking slot, then analogous to 2 a) two possibilities need to be discussed. When the time of allocation $t_{i \prime \prime \prime}^{0}=t_{i}^{0}$, the

i. The period from which the minimum of virtual payments is chosen as the actual payment in Equation (14) does not change. Same as in 2a)-i., the virtual payment of driver $i$ at that period is the same whether reporting $v_{i}$ or $v_{i}^{\prime}$, and is less than $M$.

ii. The period from which the minimum of virtual payments is chosen as the actual payment in (14) is different. It can only be that the driver is allocated at a later period $t_{i \prime \prime \prime}^{0}>t_{i}^{0}$. By Lemma 1 , virtual payments in $\left[t_{i \prime \prime \prime}^{0}, w_{i}\right]$ are invariant to valuation reporting. But fewer terms are now involved in the min operator in Equation (14), and consequently driver $i$ 's actual payment is equal to $\min _{t \in\left[t_{i \prime \prime}^{\prime \prime}, w_{i}\right]} p_{i, v}^{t} \geq \min _{t \in\left[t_{i}^{0}, w_{i}\right]} p_{i, v}^{t}=p_{i}^{a}$. The driver has no incentive to misreport a lower value than $v_{i}$.

Overall, the driver has no incentive to report a lower valuation.

Based on the above case analysis, we conclude that driver $i$ has no incentive to misreport her true valuation.

\section{Appendix C: Implementing $M\left(a^{t}, p^{t}\right)$ with Poisson arrivals}

Here we consider Poisson distribution of drivers' arrival. The mean rate of arrival $(\boldsymbol{\lambda})$ is as assumed to be 9. To maintain consistency with the analysis in Section 4, we truncate the lower limit of arrival time in the Poisson distribution at 1 and the upper limit at 20. Given $e_{i}$, generation of the a driver's latest waiting time, departure time, and valuation is the same as in sub-Section 4.1. The total number of parking slots, which are all made available at the beginning, is 100. Again, 50 simulation runs are executed.

Figure C.1 presents the number of assigned drivers over time. All drivers are immediately assigned slots upon their arrivals from the 1 st until the $8^{\text {th }}$ period, when excess supply presents. Consequently the distribution of the number of assigned drivers during this interval resembles the corresponding part of the Poisson distribution used to generate arrivals. Once the parking facility is full, the number of assigned drivers drops and equals the number of departures with capacity constraints. Similar to Figure 2a, the number of assigned drivers increases as more occupying drivers leave the parking facility in latter periods. There will be very few new arrivals near the end, and all previously waiting drivers have already been assigned. This explains drop of assigned drivers in the 19th and 20th periods. 


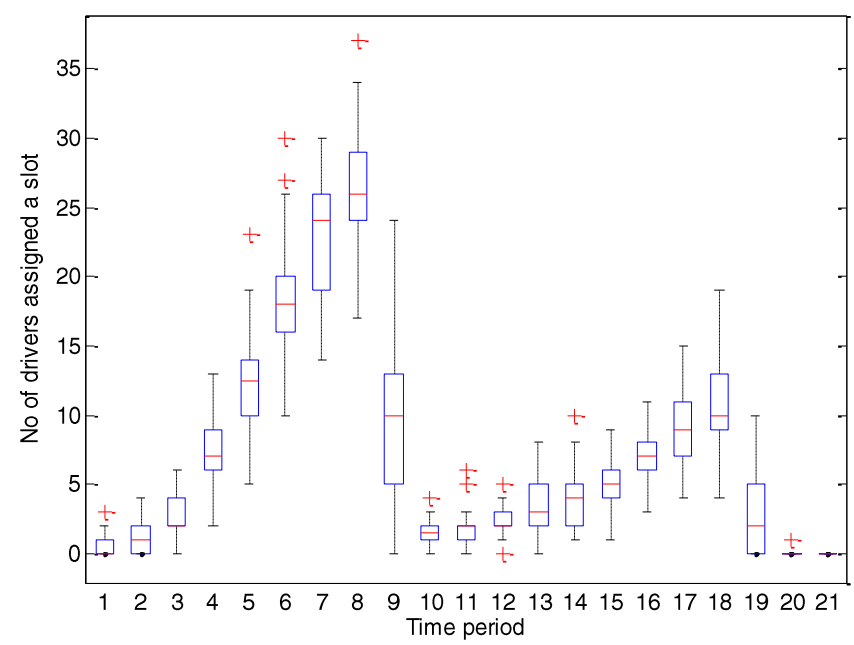

Figure C.1: Number of assigned drivers over time

The average payment per assigned driver, which is shown in Figure C.2, is similar to what we see in Figure 5a. Due to ample capacity drivers arriving before the $8^{\text {th }}$ period do not pay anything under $\boldsymbol{M}\left(\boldsymbol{a}^{\boldsymbol{t}}, \boldsymbol{p}^{\boldsymbol{t}}\right)$. Then as capacity becomes constrained positive payment persists from the $9^{\text {th }}$ to the $18^{\text {th }}$ period. After the $18^{\text {th }}$ period, payment falls back to zero, because of fewer new arrivals and more drivers vacating their occupied slots.

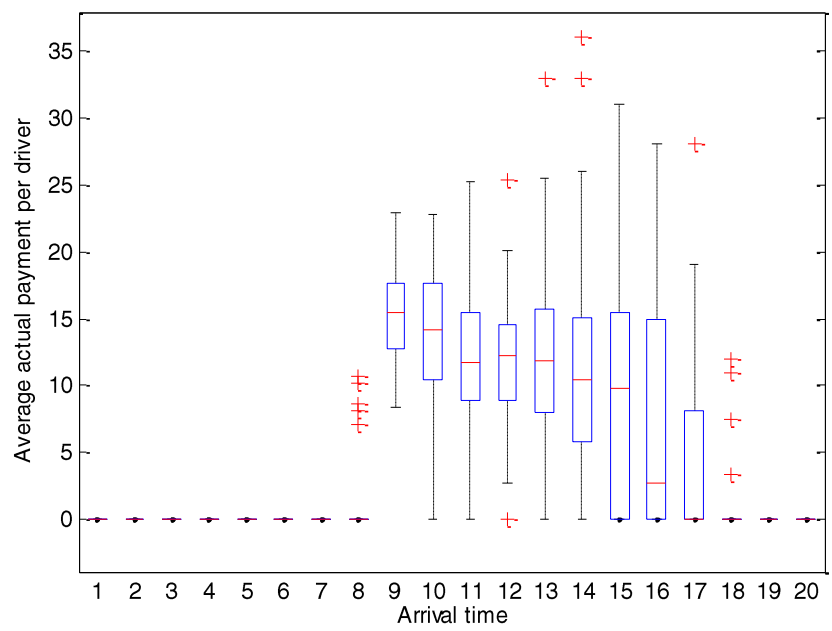

Figure C.2: Average payment per assigned drivers by their arrival time

The ratio of drivers' total payment over welfare also resembles that when a uniform arrival distribution is assumed (Figure C.3 vs. Figure 6). At the highest, about half of drivers' welfare will be transferred to the parking manager in the form of mechanism-specific payment. On average, payment captures about $18 \%$ of drivers' total welfare. 


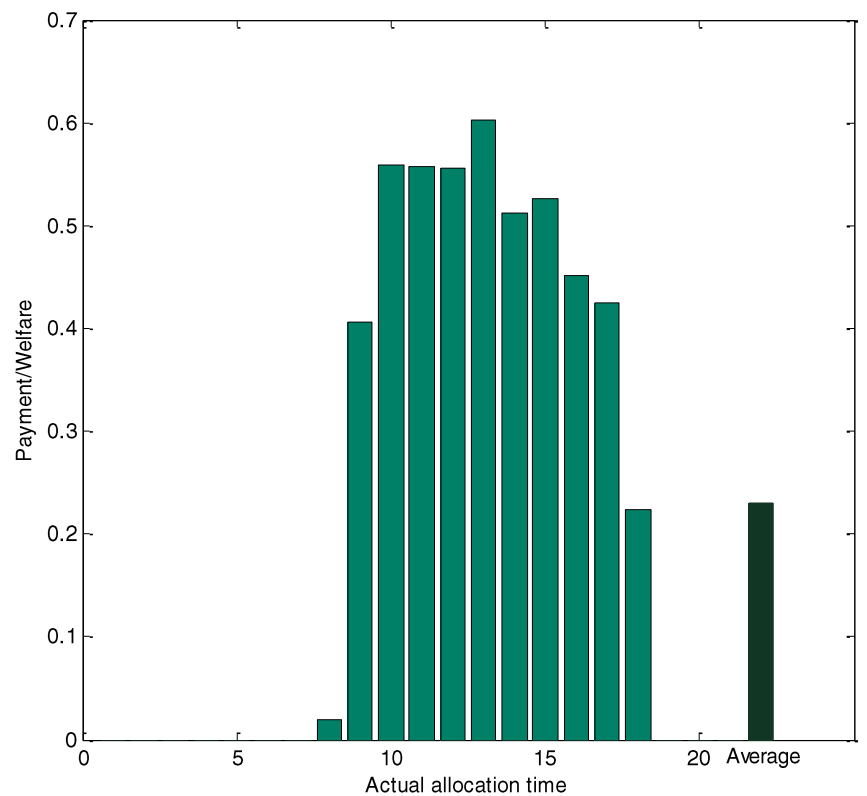

Figure C.3: Ratio of total payment over welfare gained by drivers, averaged over 50 runs

To sum up, the general insights gained from implementing $\boldsymbol{M}\left(\boldsymbol{a}^{t}, \boldsymbol{p}^{\boldsymbol{t}}\right)$ do not change whether assuming a uniform or a Poisson distribution for drivers' arrival. 